\title{
Birationally rigid varieties with a pencil of Fano double covers. II
}

\author{
Aleksandr V. Pukhlikov
}

\author{
Max-Planck-Institut für Mathematik \\ Vivatsgasse 7 \\ 53111 Bonn \\ GERMANY \\ e-mail: pukh@mpim-bonn.mpg.de \\ Steklov Institute of Mathematics \\ Gubkina 8 \\ 117966 Moscow \\ RUSSIA \\ e-mail:pukh@mi.ras.ru \\ Division of Pure Mathematics \\ Department of Mathematical Sciences \\ M\&O Building, Peach Street \\ The University of Liverpool \\ Liverpool L69 7ZL \\ ENGLAND \\ e-mail:pukh@liv.ac.uk
}

March 1, 2004

\begin{abstract}
We continue to study birational geometry of Fano fibrations $\pi: V \rightarrow \mathbb{P}^{1}$ the fibers of which are Fano double hypersurfaces of index 1. For a majority of families of this type, which do not satisfy the condition of sufficient twistedness over the base, we prove birational rigidity (in particular, it means that there are no other structures of a fibration into rationally connected varieties) and compute their groups of birational self-maps. We considerably improve the principal components of the method of maximal singularities, in the first place, the technique of counting multiplicities for the fibrations $V / \mathbb{P}^{1}$ into Fano varieties over the line.
\end{abstract}




\section{CONTENTS}

Introduction

0.1. $K^{2}$-condition and $K$-condition

0.2 . The list of varieties under consideration

0.3 . Varieties with a pencil of double spaces

0.4. Formulation of the main result

0.5. The structure of the paper

0.6. Historical remarks

0.7. Acknowledgements

1. The method of maximal singularities

1.1. Maximal singularities of linear systems

1.2. A stronger version of the Noether-Fano inequality

1.3. The self-intersection of the linear system $\Sigma$

1.4. $K^{2}$-condition and birational rigidity

1.5. The generalized $K^{2}$-condition

2. The technique of counting multiplicities

2.1. The notations and the principal claim

2.2. Proof of Proposition 2.1: counting multiplicities

2.3. Proof of Lemma 2.1

2.4. Estimating the multiplicities of a linear system:

the non-singular case

2.5. Estimating the multiplicities of a linear system:

the singular case

3. Varieties with a pencil of Fano double covers

3.1. Movable systems on the varieties of the type $((0),(2,0))$

3.2. Checking the $K$-condition

3.2.1. Varieties of the type $((0),(1,1))$

3.2.2. Varieties of the type $((2),(1,0))$

3.2.3. Varieties of the type $((2),(1,0))$

3.2.4. Varieties of the type $((2),(0,0))$

3.2.5. Varieties of the type $((3),(0,0))$

3.2.6. Varieties of the type $((1,2),(0,0))$

3.2.7. Varieties of the type $((1,1,1),(0,0)$

3.3. Proof of birational rigidity

3.4. Multiplicities of subvarieties of codimension 2

3.5. Estimating the number of lines

3.6. A method of estimating the degree

References 


\section{Introduction}

The present paper is a direct follow up of the paper [16]. We study birational geometry of higher-dimensional algebraic varieties with a pencil of Fano double covers, now without the assumption that the standard condition of sufficient twistedness over the base, that is, the $K^{2}$-condition [7,10], holds. As we pointed out in [10], if the deviation from the $K^{2}$-condition is not too big, the techniques of the method of maximal singularities still works and makes it possible to prove birational rigidity. This is the subject of the present paper: we consider Fano fibrations $V / \mathbb{P}^{1}$ which do not satisfy the $K^{2}$-condition, but which, however, present a not too strong deviation from this condition.

If the deviation from the $K^{2}$-condition oversteps a certain boundary, then the variety $V$ is no longer birationally rigid. The non-rigid and "boundary" families of fibrations $V / \mathbb{P}^{1}$ will be considered in the next paper, the third part of this research project. For that purpose we will use the improved technique developed here.

\section{$0.1 \quad K^{2}$-condition and $K$-condition}

In this paper, as in the previous paper [16], we deal with Fano fibrations $\pi: V \rightarrow \mathbb{P}^{1}$, satisfying the conditions

$$
A^{1} V=\operatorname{Pic} V=\mathbb{Z} K_{V} \oplus \mathbb{Z} F, \quad A^{2} V=\mathbb{Z} K_{V}^{2} \oplus \mathbb{Z} H_{F},
$$

where $F$ is the class of the projection $\pi, H_{F}=\left(-K_{V} \cdot F\right)$ is the hyperplane section of the fiber. Set $A_{\mathbb{R}}^{1} V=A^{1} V \otimes \mathbb{R}, A_{\mathbb{R}}^{2} V=A^{2} V \otimes \mathbb{R}$. Let

$$
A_{+}^{1} V \subset A_{\mathbb{R}}^{1} V, \quad A_{\mathrm{mov}}^{1} V \subset A_{\mathbb{R}}^{1} V, \quad A_{+}^{2} V \subset A_{\mathbb{R}}^{2} V
$$

be the closed cones, generated respectively by effective divisors, movable divisors and effective cycles of codimension two. Geometry of the fibration $V / \mathbb{P}^{1}$ is to a considerable extent determined by the position of the class $K_{V}^{2}$ with respect to the cone $A_{+}^{2} V$, and also by the position of the anticanonical class $\left(-K_{V}\right)$ with respect to the cone $A_{\text {mov }}^{1} V$. Obviously, $A_{\mathbb{R}}^{2} V=\mathbb{R} K_{V}^{2} \oplus \mathbb{R} H_{F}$, so that $A_{\mathbb{R}}^{2} V^{*}=\mathbb{R} \beta \oplus \mathbb{R} \chi$, where $\beta: A_{\mathbb{R}}^{2} V \rightarrow \mathbb{R}$ is defined by the condition $\beta\left(H_{F}\right)=1, \beta\left(K_{V}^{2}\right)=0$ and similarly $\chi\left(K_{V}^{2}\right)=1, \chi\left(H_{F}\right)=0$.

It is well known (and easy to prove, see $[7,10]$ ) that the $K^{2}$-condition

$$
K_{V}^{2} \notin \operatorname{Int} A_{+}^{2} V
$$

implies the $K$-condition:

$$
-K_{V} \notin \operatorname{Int} A_{\text {mov }}^{1} V .
$$

(There is a natural self-intersection map

$$
\begin{gathered}
\text { sq: } A_{\mathrm{mov}}^{1} V \rightarrow A_{+}^{2} V, \\
\text { sq: } z \mapsto z^{2},
\end{gathered}
$$


and it is easy to check that $\mathrm{sq}\left(\operatorname{Int} A_{\text {mov }}^{1} V\right) \subset \operatorname{Int} A_{+}^{2} V$, see Remark 1.1 in $\S 1$ below.) The present paper deals with Fano fibrations $V / \mathbb{P}^{1}$, each fiber of which is a regular Fano double hypersurface of index $1, F_{t}=\pi^{-1}(t), F_{t} \in \mathcal{F}^{\text {reg }}$, see [16], whereas the $K^{2}$-condition does not hold, that is, $K_{V}^{2} \in \operatorname{Int} A_{+}^{2} V$. The deviation from the $K^{2}$-condition is measured by the number $a \geq 0$, satisfying the formula

$$
K_{V}^{2}-a H_{F} \in \partial A_{+}^{2} V
$$

In this paper we consider mainly the fibrations $V / \mathbb{P}^{1}$, satisfying the $K$-condition. The $K$-condition is also not absolutely necessary for birational rigidity: if the deviation from the $K$-condition is not too big, the techniques of the method of maximal singularities still work and make it possible to complete the study. The families that do not satisfy the $K$-condition and also certain other families which we do not consider here and which were not considered in [16] will be studied in the next paper, the third part of this research.

\subsection{The list of varieties under consideration}

Recall the construction of varieties with a pencil of Fano double hypersurfaces, see $[9,16]$ for details. Let $\mathcal{E}=\bigoplus \mathcal{O}_{\mathbb{P}^{1}}\left(a_{i}\right)$ be a locally free sheaf of rank $M+2$, where $a_{0}=0 \leq a_{1} \leq \ldots \leq a_{M+1}, X=\mathbb{P}(\mathcal{E})$, Pic $X=\mathbb{Z} L_{X} \bigoplus \mathbb{Z} R$, where $L_{X}$ is the class of the tautological sheaf, $R$ is the class of a fiber of the natural projection $\pi_{X}: X \rightarrow \mathbb{P}^{1}$. Now the variety $V$ is realized as the double cover $\sigma: V \rightarrow Q$ of the smooth hypersurface $Q \subset X$,

$$
Q \sim m L_{X}+a_{Q} R, \quad a_{Q} \in \mathbb{Z}_{+}
$$

branched over a smooth divisor $W_{Q}=W \cap Q$, where $W \subset X$,

$$
W \sim 2 l L_{X}+2 a_{W} R, \quad a_{W} \in \mathbb{Z}_{+} .
$$

By the symbol $\pi: V \rightarrow \mathbb{P}^{1}$ we denote the natural projection, by the symbol $F_{t}$ the fiber $\pi^{-1}(t)$, sometimes omitting $t$. It is easy to see that

$$
K_{V}=-L_{V}+\left(a_{X}+a_{Q}+a_{W}-2\right) F
$$

where $L_{V}=\sigma^{*}\left(\left.L_{X}\right|_{Q}\right), a_{X}=a_{1}+\ldots+a_{M+1}$. Since the linear system $\left|L_{X}\right|$ (and therefore $\left.\left|L_{V}\right|\right)$ is free, the $K^{2}$-condition follows from the inequality

$$
\left(K_{V}^{2} \cdot L_{V}^{M-1}\right)=2 m\left(4-a_{X}-2 a_{Q}-2 a_{W}\right)+2 a_{Q} \leq 0 .
$$

In this paper we consider families that do not satisfy (11). Let us give their list. The parameters of these families are written in the following format

$$
\left(\left(a_{1}, \ldots, a_{M+1}\right),\left(a_{Q}, a_{W}\right)\right),
$$

and for brevity of notations in the set $\left(a_{1}, \ldots, a_{M+1}\right)$ we write only the non-zero entries, if there are any, otherwise we write (0): thus, for example, (1) means the set 
$(0, \ldots, 0,1),(1,1)$ means the set $(0, \ldots, 0,1,1)$, and $(0)$ stands for the set $(0, \ldots, 0)$, consisting entirely of zeros. Here is the list of varieties studied in this paper:

$$
\begin{array}{ll}
\text { 1. } & ((0),(2,0)) \\
\text { 2. } & ((0),(1,1)) \\
\text { 3. } & ((1),(0,1)) \\
\text { 4. } & ((2),(1,0)) \\
\text { 5. } & ((2),(0,0)) \\
\text { 8. } & ((3),(0,0)) \\
\text { 7. } & ((1,2),(0,0)) \\
\text { 8. } & ((1,1,1),(0,0))
\end{array}
$$

Once again we emphasize that the eight families listed above do not complete the list of varieties that do not satisfy the $K^{2}$ condition. There are seven more families not satisfying the $K$-condition, most of which are not birationally rigid, having many structures of a rationally connected fibration. These families are not considered in this paper.

\subsection{Varieties with a pencil of double spaces}

Birational rigidity of varieties $V$, fibered into double spaces of index one, was proved in the very first paper of the author, devoted to Fano fibrations, in [7], in the assumption of sufficient twistedness over the base, that is, the $K^{2}$-condition. In this paper we also consider the varieties of this type, satisfying the $K$-condition

but not the $K^{2}$-condition. Recall their construction, which is a simplification of the construction for double hypersurfaces given above (corresponding to the value $m=1)$. Let $\mathcal{E}=\bigoplus \mathcal{O}_{\mathbb{P}^{1}}\left(a_{i}\right)$ be a locally free sheaf of rank $M+1, a_{0}=0 \leq \ldots \leq a_{M}$, $X=\mathbb{P}(\mathcal{E})$ its projective bundle, then $V$ is realized as the double cover $\sigma: V \rightarrow X$, branched over a smooth hypersurface $W \subset X$,

$$
W \sim 2 M L_{X}+2 a_{W} R
$$

where $L_{X}$ is the class of the tautological sheaf, $R$ is the class of a fiber of the projection $\pi_{X}: X \rightarrow \mathbb{P}^{1}$. Obviously,

$$
K_{V}=-L_{V}+\left(a_{X}+a_{W}-2\right) F,
$$

where $L_{V}=\sigma_{*} L_{X}, F=\sigma^{*} R$ is the class of a fiber,

$$
\operatorname{Pic} V=\mathbb{Z} L_{V} \bigoplus \mathbb{Z} F
$$

$a_{X}=a_{1}+\ldots+a_{M}, a_{W} \in \mathbb{Z}_{+}$. The parameters of a family are written in the following format:

$$
\left(\left(a_{1}, \ldots, a_{M}\right), a_{W}\right)
$$


where as above if $\left(a_{1}, \ldots, a_{M}\right) \neq(0, \ldots, 0)$, then the zeros are omitted, and if $\left(a_{1}, \ldots, a_{M}\right)=(0, \ldots, 0)$, then we write simply $(0)$. A variety $V$ satisfies the $K^{2}$ condition, if the following inequality holds:

$$
\left(K_{V}^{2} \cdot L_{V}^{M-1}\right)=8-2 a_{X}-4 a_{W} \leq 0 .
$$

In the present paper we consider the families that do not satisfy (2). Here is their list:

$$
\begin{array}{ll}
1^{*} . & ((1), 1) \\
2^{*} . & ((2), 0) \\
3^{*} . & ((3), 0) \\
4^{*} . & ((1,2), 0) \\
5^{*} . & ((1,1,1), 0)
\end{array}
$$

For all the types listed above the $K$-condition is satisfied (it will be proved below). There are four families more, for which both the $K^{2}$-condition and $K$-condition are not satisfied. Their birational geometry will be studied in the next paper.

\subsection{Formulation of the main result}

For a divisor $D$ on a rationally connected variety $Y$ we set

$$
c(D, Y)=\sup \left\{\varepsilon \in \mathbb{Q}_{+} \mid D+\varepsilon K_{Y} \in A_{+}^{1} Y\right\} .
$$

For a movable linear system $\Sigma$ on a uniruled variety $V$ define the virtual threshold of canonical adjunction by the formula

$$
c_{\text {virt }}(\Sigma)=\inf _{V^{\sharp} \rightarrow V}\left\{c\left(\Sigma^{\sharp}, V^{\sharp}\right)\right\},
$$

where the infimum is taken over all birational morphisms $V^{\sharp} \rightarrow V$, where $V^{\sharp}$ is a projective model of the field $\mathbb{C}(V)$, non-singular in codimension $1, \Sigma^{\sharp}$ is the strict transform of the system $\Sigma$ on $V^{\sharp}$. The following definition of birational rigidity is equivalent to the standard one.

Definition 0.1. (i) A variety $V$ is said to be birationally superrigid, if for any movable linear system $\Sigma$ on $V$ the following equality holds:

$$
c_{\mathrm{virt}}(\Sigma)=c(\Sigma, V) \text {. }
$$

(ii) A variety $V$ (respectively, a Fano fibration $V / S$ ) is said to be birationally rigid, if for any movable linear system $\Sigma$ on $V$ there exists a birational self-map $\chi \in \operatorname{Bir} V$ (respectively, a fiber-wise self-map $\chi \in \operatorname{Bir}(V / S)$ ), satisfying the following equality:

$$
c_{\mathrm{virt}}(\Sigma)=c\left(\chi_{*} \Sigma, V\right) \text {. }
$$

Let us formulate the main result of the paper.

Theorem 1. (i) Regular varieties $V$ with a pencil of double hypersurfaces of the types $2-8$ in the list above and all varieties of the types $1^{*}-5^{*}$ with a pencil of double 
spaces are birationally superrigid and satisfy the $K$-condition: $-K_{V} \notin A_{\text {mov }}^{1} V$. The defined structure of a rationally connected fibration $V / \mathbb{P}^{1}$ is the unique non-trivial structure of a rationally connected fibration on $V$. The groups of birational and biregular self-maps of these varieties coincide:

$$
\operatorname{Bir} V=\text { Aut } V=\mathbb{Z} / 2 \mathbb{Z}
$$

(ii) The regular varieties $V$ of the type 1 (that is, the varieties of the family $((0),(2,0)))$ are birationally rigid. They have a non-trivial birational self-map, an involution $\tau \in \operatorname{Bir} V \backslash$ Aut $V$, and do not satisfy the $K$-condition. On $V$ there are exactly two non-trivial structures of a rationally connected fibration: the pencil $|F|$ of fibers of the morphism $\pi$ and its image $\left|\tau_{*} F\right|$. The group Bir $V$ consists of four elements:

$$
\operatorname{Bir} V=<\tau>\times \text { Aut } V=(\mathbb{Z} / 2 \mathbb{Z})^{\times 2} \text {. }
$$

Corollary 0.1. All varieties of the types $1-8$ and $1^{*}-5^{*}$ do not admit a structure of a rationally connected fibration over a base of dimension 2 or higher, in particular, they cannot be fibered by a rational map into rational curves or rational surfaces. Therefore they all are non-rational.

\subsection{The structure of the paper}

To prove Theorem 1, it is necessary to improve the technique of the method of maximal singularities. Such an improvement is important by itself since it extends the domain where the method works: birational rigidity is proved for far from all the natural families of Fano fibrations over $\mathbb{P}^{1}$, even in the assumption of sufficient twistedness over the base. For this reason, a bigger part of the paper is of technical character.

In $\S 1$ we carry out some preparatory work: we prove that the virtual and actual thresholds of canonical adjunction coincide provided that the $K^{2}$-condition holds and the fibers of the Fano fibration $V / \mathbb{P}^{1}$ satisfy the standard conditions (h), (v) and (vs) (see [16]). After that we make the first step in the direction of improving the technique: we show that replacing the condition $(\mathrm{h})$ by a stronger one compensates replacing the $K^{2}$-condition by a weaker one.

In $\S 2$ we improve the technique of the method of maximal singularities on the basis of a radically different idea: to compare singularities of the horizontal cycle $Z^{h}$ and of its restriction onto the fiber $F$, containing the centre of the maximal singularity. This idea is new. We use it to prove birational rigidity for the first time. Besides we give in full detail the technique of counting multiplicities: in all the previous papers [7-16] we computed the multiplicities of the cycle $Z$ of intersection of two divisors of the linear system $\Sigma$, whereas here we intersect a divisor and an irreducible subvariety of codimension 2. The computations are parallel to the divisorial case, however there are some differences: for example, only the blow ups of subvarieties of codimension 3 and higher are taken into account whereas the blow ups of subvarieties of codimension 3 play the same part as the blow ups of subvarieties of codimension 2 in the divisorial case. 
In $\S 3$ we prove Theorem 1 . First we check the $K$-condition for varieties from the lists above. After that the necessary estimates are verified for subvarieties $Y \subset F$ of an arbitrary fiber $F$ (this work was mostly carried out in the previous paper [16] and in [9]). The remaining part of the section contains an improvement of one estimate for multiplicities of subvarieties of codimension two. In order to do that, one needs to get an estimate for the number of lines (counted with multiplicities) through an arbitrary point of a fiber. Combining this improved estimate with the general theory developed in the first two sections, one could prove the coincidence of the virtual and actual thresholds of canonical adjunction for varieties deviating from the $K^{2}$-condition even stronger than those considered in this paper. These limit resources of the techniques of the method of maximal singularities will be used later.

\subsection{Historical remarks}

As soon as in [7] an effectively working technique of investigating birational geometry of Fano fibrations $V / \mathbb{P}^{1}$ satisfying the $K^{2}$-condition was developed, the "boundary" cases for which the deviation from the $K^{2}$-condition was not too high, came up as a natural object of further research. Already in [10] it was mentioned that for the particular varieties which have already been studied the $K^{2}$-condition was unnecessarily strong: the inequalities ensuring birational rigidity have a considerable amount "in store". Thus weakening this condition a little bit should not change the final result. The papers of M.M.Grinenko [3-5] and I.V.Sobolev [19,20] confirmed this idea. In [4] it was conjectured that the $K$-condition (which is weaker than the $K^{2}$-condition) is already sufficient for birational rigidity. This conjecture has recently attracted new attention [1] in connection with the attempts to study birational geometry of three-fold conic bundles (over $\mathbb{P}^{2}$ ) which do not satisfy the Sarkisov condition $[17,18]$ (this condition is an exact analog of the $K^{2}$-condition for conic bundles). However, in the papers $[19,20]$ certain varieties were successfully studied for which even the $K$-condition was not true (although the deviation from this condition was not too strong, either). And the technique used in $[19,20]$ was considerably weaker than that available today (see $\S 2$ of the present paper). This is an evidence that we do not understand which mechanisms control birational rigidity (perhaps one should speak of the "degree of birational rigidity"). On the other hand one can be optimistic concerning the prospects of studying birational geometry of Fano fibrations by means of the method of maximal singularities.

\subsection{Acknowledgements}

An essential part of this work was carried out at Max-Planck-Institut für Mathematik in Bonn in the autumn 2003. The author is very thankful to the Max-PlanckInstitut für Mathematik in Bonn for hospitality and the wonderful conditions of work. 


\section{The method of maximal singularities}

In this section we remind the main facts of the method of maximal singularities: the Noether-Fano inequality, the concepts of a maximal and a supermaximal singularities, the technique of computing the self-intersection of a movable linear system $[8,13,15]$. We prove Theorem 2 of the previous paper [16]. After that, we modify the technique for the case when the $K^{2}$-condition does not hold.

\subsection{Maximal singularities of linear systems}

Let $\Sigma \subset\left|-n K_{V}+l F\right|, l \in \mathbb{Z}_{+}$, be a movable linear system on the variety $V$. Since $l \geq 0$, we get

$$
c(\Sigma)=n
$$

whereas $n=0$ if and only if the linear system $\Sigma$ comes from the pencil $|F|$, that is, if it is pulled back from the base $\mathbb{P}^{1}$.

Remark 1.1. If the fibration $V / \mathbb{P}^{1}$ satisfies the $K^{2}$-condition, that is,

$$
K_{V}^{2} \notin \operatorname{Int} A_{+}^{2} V,
$$

then for any movable system $\Sigma \subset\left|-n K_{V}+l F\right|$ we have $l \geq 0$. Indeed, the self-intersection of the linear system $\Sigma$

$$
\left(-n K_{V}+l F\right)^{2}=n^{2} K_{V}^{2}+2 n l H_{F}
$$

is the class of an effective cycle of codimension two. By the $K^{2}$-condition this implies that $l \in \mathbb{Z}_{+}$.

Assume that the inequality

$$
c_{\text {virt }}(\Sigma)<c(\Sigma)=n,
$$

holds, that is, there is a model $V^{\sharp}$ of the variety $V$ such that

$$
c\left(\Sigma^{\sharp}, V^{\sharp}\right)<c(\Sigma, V) .
$$

Proposition 1.1. There is a prime divisor $E \subset V^{\sharp}$, satisfying the Noether-Fano inequality

$$
\nu_{E}(\Sigma)>n \cdot a(V, E)
$$

The geometric discrete valuation $\nu_{E}$ of the field of rational functions $\mathbb{C}(V)$, or any prime divisor $E^{+} \subset V^{+}$on any model $V^{+}$of the variety $V$, realizing this discrete valuation, is called a maximal singularity of the linear system $\Sigma$.

For a proof of Proposition 1.1, see any of the papers [6-8,13,15].

Note that the expression $a(V, E)$ in (3) denotes the discrepancy of $E$ with respect to the original model $V$. Thus the $\log$-pair

$$
\left(V, \frac{1}{n} \Sigma\right)
$$


is not canonical: the exceptional divisor $E$ realizes its singularity which is not canonical.

Since the linear system $\Sigma$ is movable and $\nu_{E}(\Sigma)>0$, we conclude that the centre of the discrete valuation $\nu_{E}$ on $V$ is a subvariety $B=\operatorname{centre}_{V}\left(\nu_{E}\right) \subset V$ of codimension at least two. Let

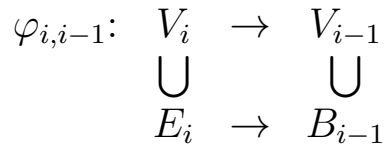

be the sequence of blow ups with irreducible centres $B_{i-1} \subset V_{i-1}$, which is determined in a unique way by the following conditions:

(1) $V_{0}=V, B_{0}=B, i=1, \ldots, K$;

(2) $B_{j}=$ centre $_{V_{j}}\left(\nu_{E}\right) \subset V_{j}, E_{j+1}=\varphi_{j+1, j}^{-1}\left(B_{j}\right)$;

(3) the valuation $\nu_{E_{K}}$ coincides with $\nu_{E}$.

In other words, the birational map

$$
V_{K}-\rightarrow V^{\sharp}
$$

is biregular at the generic point of the divisor $E_{K}$ and transforms $E_{K}$ into $E$. The symbol $\Sigma^{j}$ below means the strict transform of the linear system $\Sigma$ on $V_{j}$. Set

$$
\nu_{j}=\operatorname{mult}_{B_{j-1}} \Sigma^{j-1}, \quad \delta_{j}=\operatorname{codim} B_{j-1}-1 .
$$

On the set of exceptional divisors

$$
\left\{E_{1}, \ldots, E_{K}\right\}
$$

we define in the usual way $[6,8,13]$ an oriented graph structure: an oriented edge (an arrow) goes from $E_{i}$ to $E_{j}$, if and only if $i>j$ and

$$
B_{i-1} \subset E_{j}^{i-1}
$$

which is denoted as $i \rightarrow j$. As usual, for $i>j$ set

$$
p_{i j}=\sharp\left\{\text { the paths from } E_{i} \text { to } E_{j}\right\} \geq 1 \text {, }
$$

$p_{i i}=1$ by definition. Set $p_{i}=p_{K i}$. The Noether-Fano inequality takes the traditional form

$$
\sum_{i=1}^{K} p_{i} \nu_{i}>n \sum_{i=1}^{K} p_{i} \delta_{i} .
$$

\subsection{A stronger version of the Noether-Fano inequality}

Now assume that the general fiber $F=F_{t}$ of the fiber space $V / \mathbb{P}^{1}$ admits no movable linear system with a maximal singularity, that is, for any movable system $\Sigma_{F} \subset$ $\left|n H_{F}\right|=\left|-n K_{F}\right|$ and any geometric discrete valuation $\nu_{E^{*}}$ the inequality

$$
\nu_{E^{*}}\left(\Sigma_{F}\right) \leq n a\left(E^{*}\right)
$$


holds.

Proposition 1.2. The centre $B$ of the maximal singularity $\nu_{E}$ on $V$ is contained in some fiber $\pi^{-1}(t)=F_{t}, t \in \mathbb{P}^{1}$.

Proof. Assume the converse: $\pi(B)=\mathbb{P}^{1}$. Let $F \subset V$ be a fiber of general position. It is easy to see that the restriction $\Sigma_{F}=\left.\Sigma\right|_{F}$ of the linear system $\Sigma$ onto $F$ is a movable linear system $\Sigma_{F} \subset\left|n H_{F}\right|$ with a maximal singularity $\nu_{E^{*}}=\left.\nu_{E}\right|_{F}$. The simplest way to define this singularity is to restrict the sequence of blow ups (4) onto the fiber $F$ and note that the discrepancy remains the same:

$$
a\left(\left.E\right|_{F}, F\right)=a(E, V)
$$

- precisely for the reason that $B$ covers the base. The centre of the valuation $\left.\nu_{E}\right|_{F}$ is $B_{F}=B \cap F$. However, this conclusion contradicts the assumption above. Therefore, $\pi(B) \neq \mathbb{P}^{1}$. Q.E.D. for the proposition.

Let $\mathcal{M}=\left\{T_{1}, \ldots, T_{k}\right\}$ be the set of all prime divisors on $V^{\sharp}$, satisfying the Noether-Fano inequality (see Proposition 1.1). As we have just proved, the centre $B_{E}=\operatorname{centre}\left(\nu_{E}\right)$ of each maximal singularity $E \in \mathcal{M}$ is contained in some fiber $F_{t}$. The set $\mathcal{M}$ is finite (since the model $V^{\sharp}$ is fixed), so that there is at most finite set of points $t \in \mathbb{P}^{1}$, the fibers $F_{t}$ over which contain the centres of maximal singularities. Set $\mathcal{M}_{t}=\left\{E \in \mathcal{M} \mid B_{E} \subset F_{t}\right\}$,

$$
e(E)=\nu_{E}(\Sigma)-n a(E, V)>0
$$

for $E \in \mathcal{M}$. Recall that $\Sigma \subset\left|-n K_{V}+l F\right|, l \in \mathbb{Z}_{+}$.

Proposition 1.3. The following inequality holds:

$$
\sum_{t \in \mathbb{P}^{1}} \max _{\left\{E \in \mathcal{M}_{t}\right\}} \frac{e(E)}{\nu_{E}\left(F_{t}\right)}>l
$$

Proof. Let $D^{\sharp} \in \Sigma^{\sharp}$ be a general divisor, that is, the strict transform on $V^{\sharp}$ of a divisor $D \in \Sigma$ of general position. By assumption, the linear system

$$
\left|D^{\sharp}+n K_{V^{\sharp}}\right|
$$

is empty. Therefore the linear system

$$
\left|l F-\sum_{E \in \mathcal{M}} e(E) E\right|
$$

is empty, too. On the other hand, by construction for $E \in \mathcal{M}_{t}$ the divisor

$$
F_{t}-\nu_{E}\left(F_{t}\right) E
$$

is effective, so that the divisor

$$
\sum_{t \in \mathbb{P}_{1}}\left[\left(\max _{\left\{E \in \mathcal{M}_{t}\right\}} \frac{e(E)}{\nu_{E}\left(F_{T}\right)}\right) F_{t}-\sum_{E \in \mathcal{M}_{t}} e(E) E\right]
$$

is also effective. This immediately implies the inequality (5). Q.E.D. for the proposition. 


\subsection{The self-intersection of the linear system $\Sigma$}

Let $D_{i} \in \Sigma, i=1,2$, be general divisors, so that the closed set $D_{1} \cap D_{2}$ is of codimension two. Let

$$
Z=\left(D_{1} \circ D_{2}\right)=Z^{v}+Z^{h}
$$

be the decomposition of the algebraic cycle of the scheme-theoretic intersection of these divisors into the vertical $\left(Z^{v}\right)$ and horizontal $\left(Z^{h}\right)$ parts. For the cycle $Z^{v}$ we have a further decomposition

$$
Z^{v}=\sum_{t \in \mathbb{P}^{1}} Z_{t}^{v}, \quad \operatorname{Supp} Z_{t}^{v} \subset F_{t} .
$$

Let $E \in \mathcal{M}_{t} \subset \mathcal{M}$ be a maximal singularity. Fix $t$ and $E$ and apply the technique of counting multiplicities, developed in $[8,10,13]$, to the cycle $Z_{t}^{v}+Z^{h}$. We assume that $t$ and $E$ are fixed throughout this subsection, so that we write below $F, Z^{v}, e$, $B$ instead of $F_{t}, Z_{t}^{v}, e(E), B_{E}=\operatorname{centre}(E)$, respectively.

Lemma 1.1. The following estimate holds:

$$
\operatorname{codim}_{F} B \geq 2
$$

Proof. Assume the converse: $B \subset F$ is a prime divisor. Let $D \in \Sigma$ be a general divisor, $D_{F}$ its restriction onto $F$. By the Noether-Fano inequality mult $_{B} D>n$, so that

$$
D_{F}=\alpha B+D^{\sharp},
$$

where $\alpha>n$ and $D^{\sharp}$ is an effective divisor on $F$. However,

$$
D_{F} \sim n H_{F},
$$

which gives an immediate contradiction. Q.E.D. for the lemma.

Now consider the sequence of blow ups (4) associated with the discrete valuation $E$. We use the notations of Sec. 1.1. The strict transforms $\left(Z^{h}\right)^{j},\left(Z^{v}\right)^{j}$ of the cycles $Z^{h}, Z^{v}$ on $V_{j}$ are well defined. By the symbol $F^{j}$ we denote the strict transform of the fiber $F$ on $V_{j}$. Set

$$
N=\max \left\{i \mid B_{i-1} \subset F^{i-1}\right\} .
$$

It is easy to see that $\varphi_{i, i-1}\left(B_{i}\right)=B_{i-1}$ for any $i=1, \ldots, K-1$, so that the codimensions codim $B_{i}$ do not increase. Set

$$
L=\max \left\{i \mid \operatorname{codim} B_{i-1} \geq 3\right\} \leq K .
$$

We use also the following notations: for $i \in\{1, \ldots, L\}$

$$
m_{i}^{h}=\operatorname{mult}_{B_{i-1}}\left(Z^{h}\right)^{i-1}, \quad m_{i}^{v}=\operatorname{mult}_{B_{i-1}}\left(Z^{v}\right)^{i-1},
$$

where $m_{i}^{h(v)} \leq m_{i-1}^{h(v)}$ for $i=2, \ldots, L$.

Set also

$$
\mu_{i}=\operatorname{mult}_{B_{i-1}} F^{i-1} .
$$


Obviously, $\mu_{i}=0$ for $i \geq N+1$. The more so, $m_{i}^{v}=0$ for $i \geq N+1$ (if $N<L$ ). Using the symbol $p_{i}$, as above, for the number of paths in the graph $\Gamma$ of the resolution (4) from the vertex $E=E_{K}$ to $E_{i}$, we get

Proposition 1.4. The following inequality holds:

$$
\sum_{i=1}^{L} p_{i} m_{i}^{h}+\sum_{i=1}^{\min \{N, L\}} p_{i} m_{i}^{v} \geq \sum_{i=1}^{K} p_{i} \nu_{i}^{2} \geq \frac{\left(n \sum_{i=1}^{K} p_{i} \delta_{i}+e\right)^{2}}{\sum_{i=1}^{K} p_{i}}
$$

Proof is obtained by applying the technique of counting multiplicities $[8,10,13]$ combined with the condition

$$
\sum_{i=1}^{K} p_{i} \nu_{i}=n \sum_{i=1}^{K} p_{i} \delta_{i}+e,
$$

where $e>0$. Here we do not repeat these standard arguments, just referring to [10].

\section{$1.4 \quad K^{2}$-condition and birational rigidity}

As a first example of using the technique described above let us prove Theorem 2 of the previous paper [16]. The arguments below follow the lines of the proof of particular cases of this theorem, given in $[7,10]$ for certain special families of Fano fiber spaces. Here we consider the general case.

Assume that the fiber space $V / \mathbb{P}^{1}$ satisfies the $K^{2}$-condition: $K_{V}^{2} \notin \operatorname{Int} A_{+}^{2} V$. Then for some $\alpha \in \mathbb{Z}_{+}$the following relation holds:

$$
Z^{h} \sim n^{2} K_{V}^{2}+\alpha H_{F}
$$

so that

$$
\operatorname{deg} Z^{v}=\sum_{t \in \mathbb{P}^{1}} \operatorname{deg} Z_{t}^{v} \leq(2 n \operatorname{deg} V) l .
$$

Proposition 1.5. For some point $t \in \mathbb{P}^{1}$ there is a maximal singularity $E \in \mathcal{M}_{t}$, satisfying the estimate

$$
e(E)>\frac{\nu_{E}\left(F_{t}\right)}{2 n \operatorname{deg} V} \operatorname{deg} Z_{t}^{v}
$$

Following $[7,10]$, we call the singularity $E$, satisfying the inequality (8) , a supermaximal singularity.

Proof of Proposition 1.5: it is sufficient to compare the inequalities (5) and (71). Q.E.D.

Now in the notations of subsection 1.3 set

$$
\Sigma_{l}=\sum_{i=1}^{L} p_{i}, \quad \Sigma_{u}=\sum_{i=L+1}^{K} p_{i}, \quad \Sigma_{f}=\sum_{i=2}^{\min \{N, L\}} p_{i} .
$$


Note that

$$
\nu_{E}(F)=\sum_{i=1}^{N} p_{i} \mu_{i} \leq p_{1} \mu_{1}+\mu_{2} \Sigma_{f}
$$

by definition of the multiplicities $\mu_{i}$. Obviously,

$$
m_{i}^{h} \leq m_{h}=m_{1}^{h}=\operatorname{mult}_{B} Z^{h} .
$$

Set also

$$
d_{h}=\operatorname{deg} Z^{h}, \quad d_{v}=\operatorname{deg} Z_{t}^{v}
$$

and introduce the coefficients

$$
k_{h}=\frac{m_{h}}{d_{h}} \operatorname{deg} V, \quad k_{v}=\frac{\operatorname{deg} V}{\nu_{E}(F) d_{v}} \sum_{i=1}^{\min \{N, L\}} p_{i} m_{i}^{v} .
$$

Now Proposition 1.5 implies

Corollary 1.1. The following estimate holds:

$$
\left(4-k_{h}\right) \Sigma_{l}\left(\Sigma_{l}+\Sigma_{u}\right) n^{2}+\Sigma_{u}^{2} n+e^{2}+2\left(2-k_{v}\right) \Sigma_{l} n e+2\left(1-k_{v}\right) \Sigma_{u} n e<0 .
$$

Proof. In the inequality (6) replace $m_{i}^{h}$ by $m_{h}=k_{h} d_{h} / \operatorname{deg} V=k_{h} n^{2}$, the numbers $\delta_{i}$ for $i \leq L$ by 2 , which could only make the inequality sharper. Now taking into account the definition of the coefficient $k_{v}$ after easy computations we get

$$
\begin{gathered}
\left(4-k_{h}\right) \Sigma_{l}\left(\Sigma_{l}+\Sigma_{u}\right) n^{2}+\left(n \Sigma_{u}+e\right)^{2}+4 \Sigma_{l} e n- \\
-k_{v} \frac{d_{v} \nu_{E}(F)}{\operatorname{deg} V}\left(\Sigma_{l}+\Sigma_{u}\right) \leq 0 .
\end{gathered}
$$

Taking into consideration the definition of a supermaximal singularity (Proposition 1.5), replace $d_{v} \nu_{E}(F)$ by $2 n e \operatorname{deg} V$. This makes our inequality a strict one and we obtain exactly what we claimed. Q.E.D. for the corollary.

Now assume that the fiber space $V / \mathbb{P}^{1}$ satisfies

- the condition (v) of the paper [16], that is, for any irreducible vertical subvariety $Y$ of codimension 2, $Y \subset \pi^{-1}(t)=F_{t}$, and any smooth point $o \in F_{t}$ the estimate

$$
\frac{\text { mult }_{o}}{\operatorname{deg}} Y \leq \frac{2}{\operatorname{deg} V}
$$

holds;

- the condition (vs) of the paper [16], that is, for any vertical subvariety $Y \subset F_{t}$ of codimension 2 (with respect to $V$, that is, for a prime divisor on $F_{t}$ ), a singular point $o \in F_{t}$ and an infinitely near point $x \in \widetilde{F}_{t}$, where $\varphi: \widetilde{F}_{t} \rightarrow F_{t}$ is a blow up of the point $o, \varphi(x)=o, \widetilde{Y} \subset \widetilde{F}_{t}$ the strict transform of the subvariety $Y$ on $\widetilde{F}_{t}$, the following estimates hold:

$$
\frac{\text { mult }_{o}}{\operatorname{deg}} Y \leq \frac{4}{\operatorname{deg} V}, \quad \frac{\operatorname{mult}_{x} \widetilde{Y}}{\operatorname{deg} Y} \leq \frac{2}{\operatorname{deg} V}
$$


- the condition (h) of the paper [16], that is, for any horizontal subvariety $Y$ of codimension 2 and a point $o \in Y$ the estimate

$$
\frac{\operatorname{mult}_{o}}{\operatorname{deg}} Y \leq \frac{4}{\operatorname{deg} V}
$$

holds.

In these assumptions we get

Lemma 1.2. The following estimates hold: $k_{h} \leq 4, k_{v} \leq 2$.

Proof. The first inequality follows directly from the definition of the number $k_{h}$ and the condition (h), which is by assumption satisfied for the fiber space $V / \mathbb{P}^{1}$. Let us prove the second inequality. We get

$$
\left(\frac{d_{v}}{\operatorname{deg} V}\right) k_{v}=\frac{\sum_{i=1}^{\min \{N, L\}} p_{i} m_{i}^{v}}{\sum_{i=1}^{N} p_{i} \mu_{i}} \leq \frac{p_{1} m_{1}^{v}+\Sigma_{f} m_{2}^{v}}{p_{1} \mu_{1}+\Sigma_{f}}
$$

If $\operatorname{dim} B \geq 1$ or $B=o \in F$ is a non-singular point of the fiber, then $\mu_{1}=\ldots=$ $\mu_{N}=1$ and by the condition (v)

$$
m_{2}^{v} \leq m_{1}^{v} \leq 2 \frac{d_{v}}{\operatorname{deg} V}
$$

which immediately implies the inequality $k_{v} \leq 2$. If $B=o \in F$ is a singular point of the fiber, then $\mu_{1} \geq 2$ and by the condition (vs)

$$
m_{1}^{v} \leq \frac{4 d_{v}}{\operatorname{deg} V}, \quad m_{2}^{v} \leq \frac{2 d_{v}}{\operatorname{deg} V}
$$

whence we get again that $k_{v} \leq 2$. Q.E.D. for the lemma.

Recall the claim of Theorem 2 of the paper [16]:

Assume that a smooth standard Fano fiber space $V / \mathbb{P}^{1}$ satisfies the $K^{2}$-condition and the conditions $(\mathrm{v})$, (vs) and $(\mathrm{h})$. Then $V / \mathbb{P}^{1}$ is birationally superrigid.

Let us complete the proof of this theorem. Assume the converse. Then Corollary 1.1 and Lemma 1.2 give us the inequality

$$
\Sigma_{u}^{2} n^{2}-2 \Sigma_{u} n e+e^{2}<0 .
$$

This is, however, impossible. We get a contradiction. Q.E.D. for Theorem 2 of the paper [16]. 


\subsection{The generalized $K^{2}$-condition}

The methods developed in $[7,10]$ and reproduced above in the general form work well for Fano fiber spaces $V / \mathbb{P}^{1}$ which do not satisfy the $K^{2}$-condition. If the deviation from the $K^{2}$-condition is not too great then the technique of Sec. 1.4 is still effective and requires only a slight modification. First of all, one should be able to measure the deviation from the $K^{2}$-condition.

Definition 1.1. A standard Fano fiber space $V / \mathbb{P}^{1}$ satisfies the generalized $K^{2}$ condition of depth $\varepsilon \geq 0$, if

$$
K_{V}^{2}-\varepsilon H_{F} \notin \operatorname{Int} A_{+}^{2} V
$$

As mentioned in [10], for effective cycles of codimension 2 on the natural Fano varieties sharper estimates on the multiplicities are satisfied than one needs to prove birational rigidity. However, for Fano fiber spaces that do not satisfy the $K^{2}$-condition these estimates turn out to be useful.

Definition 1.2. A standard Fano fibration $V / \mathbb{P}^{1}$ satisfies the generalized condition (h) of depth $\delta \geq 0$, if for any horizontal subvariety $Y \subset V$ of codimension two and an arbitrary point $o \in Y$ the following inequality holds:

$$
\frac{\text { mult }_{o}}{\operatorname{deg}} Y \leq \frac{4-\delta}{\operatorname{deg} V}
$$

Starting from this moment and throughout this section we assume that the fiber space $V / \mathbb{P}^{1}$ satisfies the generalized $K^{2}$-condition of depth $\varepsilon \geq 0$ and the generalized condition (h) of depth $\delta \geq 0$. Besides, we assume that the conditions (v) and (vs) are satisfied (in their normal form). Fix a movable linear system $\Sigma \subset\left|-n K_{V}+l F\right|$ with $l \in \mathbb{Z}_{+}$and assume that $c_{\mathrm{virt}}(\Sigma)<n$. For the horizontal part of the self-intersection of the linear system $\Sigma$ we get

$$
Z^{h} \sim n^{2} K_{V}^{2}+\alpha H_{F}
$$

where the coefficient $\alpha \in \mathbb{Z}$ satisfies the inequality

$$
\alpha \geq-\varepsilon n^{2} .
$$

Therefore, for the vertical component we get

$$
Z^{v} \sim(2 n l-\alpha) H_{F}
$$

whence

$$
\operatorname{deg} Z^{v}=\sum_{t \in \mathbb{P}^{1}} \operatorname{deg} Z_{t}^{v} \leq\left(2 n l+\varepsilon n^{2}\right) \operatorname{deg} V .
$$

Proposition 1.6. For some point $t \in \mathbb{P}^{1}$ there exists a maximal singularity $E \in \mathcal{M}_{t} \neq \emptyset$, satisfying the estimate

$$
e(E)>\frac{\nu_{E}\left(F_{t}\right)}{2}\left(\frac{\operatorname{deg} Z_{t}^{v}}{n \operatorname{deg} V}-\varepsilon n\right)
$$


Remark 1.2. For $\varepsilon=0$ we get Proposition 1.5.

Proof of Proposition 1.6. Compare the inequalities (5) and (10). Replacing the number $l$ in the right-hand side of the inequality (10) by the left-hand side of the inequality (5), we get

$$
\sum_{t \in \mathbb{P}^{1}}\left[\operatorname{deg} Z_{t}^{v}-2 n \operatorname{deg} V \max _{\left\{E \in \mathcal{M}_{t}\right\}} \frac{e(E)}{\nu_{E}\left(F_{t}\right)}\right]<\varepsilon n^{2} \operatorname{deg} V,
$$

which immediately implies our claim. Q.E.D. for Proposition 1.6.

Remark 1.3. If there are a few maximal singularities the centres of which lie in the fibers over distinct points $t_{1}, \ldots, t_{k}$ then the claim of Proposition 1.6 can be improved: there is a maximal singularity $E \in \mathcal{M}_{t}, t \in\left\{t_{1}, \ldots, t_{k}\right\}$ satisfying the estimate

$$
e(E)>\frac{\nu_{E}\left(F_{t}\right)}{2}\left(\frac{\operatorname{deg} Z_{t}^{v}}{n \operatorname{deg} V}-\frac{\varepsilon n}{k}\right)
$$

However, this improvement is hardly useful, since to prove birational rigidity, that is, to realize the full scheme of the method of maximal singularities, the worst case should be considered.

Now we argue as in the proof of Corollary 1.1 with the only difference: the expression $d_{v} \nu_{E}(F)$ in the inequality (91) is replaced by the expression

$$
\left(2 n e+\varepsilon n^{2} \nu_{E}(F)\right) \operatorname{deg} V,
$$

which makes the inequality sharp. Taking into account that the conditions (h), (v) and (vs) still hold, so that the claim of Lemma 1.2 is true, we get the inequality

$$
\left(\left(4-k_{h}\right) \Sigma_{l}-k_{v} \varepsilon \nu_{E}(F)\right)\left(\Sigma_{l}+\Sigma_{u}\right) n^{2}+\left(n \Sigma_{u}-e\right)^{2}<0 .
$$

By the definition of the number $k_{v}$ we get

$$
k_{v} \nu_{E}(F)=\operatorname{deg} V \sum_{i=1}^{\min \{N, L\}} p_{i} \frac{m_{i}^{v}}{d_{v}} \leq 2 \Sigma_{l} .
$$

Therefore, the following inequality holds:

$$
\left(4-k_{h}-2 \varepsilon\right) \Sigma_{l}\left(\Sigma_{l}+\Sigma_{u}\right) n^{2}+\left(n \Sigma_{u}-e\right)^{2}<0 .
$$

Now recall that by the generalized condition (h) of depth $\delta \geq 0$ the coefficient $k_{h}$ satisfies the estimate

$$
k_{h} \leq 4-\delta
$$

This immediately implies

Proposition 1.7. If $\delta \geq 2 \varepsilon$, then the equality

$$
c_{\text {virt }}(\Sigma)=c(\Sigma)=n
$$

holds. In particular, if for any movable linear system $\Sigma \subset\left|-n K_{V}+l F\right|$ we have $l \in \mathbb{Z}_{+}$, then the fiber space $V / \mathbb{P}^{1}$ is birationally superrigid. 


\section{The technique of counting multiplicities}

The aim of this section is to sharpen the inequality (12) and the claim of Proposition 1.7. For this purpose we take into consideration the dimensions of the blown up subvarieties $B_{i-1}$ : the smaller is the dimension of the centre of a blow up, the higher is the discrepancy of the exceptional divisor and, therefore, the better is the estimate for the multiplicity $\nu_{E}(\Sigma)$. On the other hand, the only working method of getting an upper bound for the singularities of the horizontal cycle $Z^{h}$ is to restrict $Z^{h}$ onto the fiber $F$ and estimate the singularities of the effective cycle $\left(Z^{h} \circ F\right)$ of codimension two on $F$. This method was used above; also in the papers $[7,10,16]$ the singularities of the cycle $Z^{h}$ were estimated in this way. However, up to this day we never took into account the input of the subvarieties $B_{i-1}$ of codimension three (with respect to $V$ ), lying in the strict transform $F^{i-1}$ of the fiber $F$ (provided they exist). That is what we do below. As a result, we obtain estimates which make it possible to exclude a maximal singularity even in cases when the deviation from the $K^{2}$-condition is essential: it is sufficient that the generalized $K^{2}$-condition of depth 2 holds.

\subsection{The notations and the principal claim}

We go on studying the movable linear system $\Sigma \subset\left|-n K_{V}+l F\right|, l \in \mathbb{Z}_{+}$, satisfying the inequality

$$
c_{\mathrm{virt}}(\Sigma)<c(\Sigma)=n
$$

All notations of $\S 1$ are valid. However, if in subsections 1.4 and 1.5 we treated all blow ups of subvarieties of codimension three and higher in the same way, now we argue in a more refined way. Set

$$
\begin{gathered}
J_{s}=\left\{i \mid 1 \leq i \leq K, \quad \operatorname{codim} B_{i-1} \geq 4\right\}, \\
J_{m}=\left\{i \mid 1 \leq i \leq K, \quad \operatorname{codim} B_{i-1}=3\right\}, \\
J_{u}=\{i \mid L+1 \leq i \leq K\}, \quad J_{l}=J_{s} \cup J_{m} .
\end{gathered}
$$

In its turn, let us break the set $J_{m}$ into two disjoint subsets, $J_{m}=J_{m}^{+} \amalg J_{m}^{-}$, where

$$
J_{m}^{+}=\left\{i \in J_{m} \mid B_{i-1} \subset F^{i-1}\right\},
$$

$J_{m}^{-}=J_{m} \backslash J_{m}^{+}=\left\{i \in J_{m} \mid B_{i-1} \not \subset F^{i-1}\right\}$. It might well turn out that the set $J_{m}^{+}$or $J_{m}^{-}$(or the whole set $J_{m}$ ) is empty. Set furthermore

$$
\Sigma_{s}=\sum_{i \in J_{s}} p_{i}, \quad \Sigma_{m}^{ \pm}=\sum_{i \in J_{m}^{ \pm}} p_{i}, \quad \Sigma_{m}=\Sigma_{m}^{+}+\Sigma_{m}^{-},
$$

whereas the symbol $\Sigma_{u}$ retains its previous meaning. In the notations of Sec. 1.4 we get $\Sigma_{l}=\Sigma_{s}+\Sigma_{m}$. Now the inequality (6) can be rewritten as

$$
\sum_{i \in J_{l}} p_{i} m_{i}^{h}+\sum_{i \in J_{S} \cup J_{m}^{+}} p_{i} m_{i}^{v} \geq \frac{\left(\left(3 \Sigma_{s}+2 \Sigma_{m}+\Sigma_{u}\right) n+e\right)^{2}}{\Sigma_{s}+\Sigma_{m}+\Sigma_{u}}
$$


Recall that $\mu_{i}=$ mult $_{B_{i-1}} F^{i-1}$, where $\mu_{i}=1$ for $i \geq 2$ and for $\mu_{1}$ there are two possible cases: $\mu_{1}=1$ or $\mu_{1}=2$.

Proposition 2.1. The following estimate holds:

$$
\sum_{i \in J_{s} \cup J_{m}^{+}} \mu_{i} p_{i} m_{i}^{h} \leq p_{1} \operatorname{mult}_{B}\left(Z^{h} \circ F\right)+\left(\Sigma_{s}-p_{1}\right) \operatorname{mult}_{B_{1}}\left(Z^{h} \circ F\right)^{1} .
$$

In particular,

$$
\sum_{i \in J_{s} \cup J_{m}^{+}} \mu_{i} p_{i} m_{i}^{h} \leq \Sigma_{s} \operatorname{mult}_{B}\left(Z^{h} \circ F\right) .
$$

\subsection{Proof of Proposition 2.1: counting multiplicities}

To begin with, let us consider the following general situation. Let $Y \subset V$ be an irreducible horizontal subvariety of codimension two, $Y^{i} \subset V_{i}$ its strict transform,

$$
m_{Y}(i)=\operatorname{mult}_{B_{i-1}} Y^{i-1}
$$

the corresponding multiplicity. Set $Y_{F}=(Y \circ F)$. This is an effective class of codimension two in the fiber $F$. Let $Y_{F}^{i} \subset V_{i}$ be its strict transform and

$$
m_{Y, F}(i)=\operatorname{mult}_{B_{i-1}} Y^{i-1}
$$

Since the support of the cycle $Y_{F}$ is contained in the fiber $F$, the numbers $m_{Y, F}(i)$ vanish for $i \in J_{m}^{-}$.

Lemma 2.1. The following estimate holds:

$$
\sum_{i \in J_{s} \cup J_{m}^{+}} p_{i} m_{Y}(i) \mu_{i} \leq \sum_{i \in J_{s}} p_{i} m_{Y, F}(i) .
$$

Before we start to prove it, recall some facts which follow immediately from elementary intersection theory [21]. There are no convenient references because here we consider the case when a divisor intersects a subvariety, whereas in $[8,13]$ the case of two divisors was treated. Let $X$ be an arbitrary smooth variety, $B \subset X$, $B \not \subset \operatorname{Sing} X$, an irreducible subvariety of codimension $\geq 2, \sigma_{B}: X(B) \rightarrow X$ its blow up, $E(B)=\sigma_{B}^{-1}(B)$ the exceptional divisor. Let

$$
Z=\sum m_{i} Z_{i}, \quad Z_{i} \subset E(B),
$$

be a cycle of dimension $k, k \geq \operatorname{dim} B$. We define the degree of the cycle $Z$, setting

$$
\operatorname{deg} Z=\sum_{i} m_{i} \operatorname{deg}\left(Z_{i} \bigcap \sigma_{B}^{-1}(b)\right)
$$

where $b \in B$ is a point of general position, $\sigma_{B}^{-1}(b) \cong \mathbb{P}^{\text {codim } B-1}$ and the degree in the right-hand side is the usual degree in the projective space. 
Note that $\operatorname{deg} Z_{i}=0$ if and only if $\sigma_{B}\left(Z_{i}\right)$ is a proper closed subset of the subvariety $B$.

Now let $D$ be a prime Weil divisor on $X, Y \subset X$ an irreducible subvariety of dimension $l \leq \operatorname{dim} X-1$. Assume that $Y \not \subset D$ and that $\operatorname{dim} B \leq l-1$. The strict transforms of the divisor $D$ and the subvariety $Y$ on $X(B)$ are denoted by the symbols $D^{B}$ and $Y^{B}$, respectively.

Lemma 2.2. (i) Assume that $\operatorname{dim} B \leq l-2$. Then

$$
D^{B} \circ Y^{B}=(D \circ Y)^{B}+Z,
$$

where $\circ$ means the operation of taking the algebraic cycle of the scheme-theoretic intersection, $\operatorname{Supp} Z \subset E(B)$ and

$$
\operatorname{mult}_{B}(D \circ Y)=\operatorname{mult}_{B} D \cdot \operatorname{mult}_{B} Y+\operatorname{deg} Z .
$$

(ii) Assume that $\operatorname{dim} B=l-1$. Then

$$
D^{B} \circ Y^{B}=Z+Z_{1},
$$

where $\operatorname{Supp} Z \subset E(B)$, Supp $\sigma_{B}\left(Z_{1}\right)$ does not contain $B$ and

$$
D \circ Y=\left[\left(\operatorname{mult}_{B} D\right)\left(\operatorname{mult}_{B} Y\right)+\operatorname{deg} Z\right] B+\left(\sigma_{B}\right)_{*} Z_{1} .
$$

Proof is easily obtained by means of the standard intersection theory [21].

\subsection{Proof of Lemma 2.1}

Let us construct a sequence of effective cycles of codimension three on the varieties $V_{i}$, setting

$$
\begin{aligned}
Y \circ F & =Z_{0}\left(=Y_{F}\right), \\
Y^{1} \circ F^{1} & =Z_{0}^{1}+Z_{1}, \\
\vdots & \\
Y^{i} \circ F^{i} & =\left(Y^{i-1} \circ F^{i-1}\right)^{i}+Z_{i}, \\
\vdots &
\end{aligned}
$$

$i \in J_{s}$, where $\operatorname{Supp} Z_{i} \subset E_{i}$. Thus for any $i \in J_{s}$ we get:

$$
Y^{i} \circ F^{i}=Y_{F}^{i}+Z_{1}^{i}+\ldots+Z_{i-1}^{i}+Z_{i} .
$$

For any $j>i, j \in J_{s}$ set

$$
m_{i, j}=\operatorname{mult}_{B_{j-1}}\left(Z_{i}^{j-1}\right)
$$

(the multiplicity of an irreducible subvariety along a smaller subvariety is understood in the usual sense; for an arbitrary cycle we extend the multiplicity by linearity). 
Now set $d_{i}=\operatorname{deg} Z_{i}$. We get the following system of equalities:

$$
\begin{aligned}
& m_{Y}(1) \mu_{1}+d_{1}=m_{Y, F}(1) \\
& m_{Y}(2) \mu_{2}+d_{2}=m_{Y, F}(2)+m_{1,2}, \\
& \vdots \\
& m_{Y}(i) \mu_{i}+d_{i}=m_{Y, F}(i)+m_{1, i}+\ldots+m_{i-1, i}
\end{aligned}
$$

for all $i \in J_{s}$. Setting $S=\max \left\{i \in J_{s}\right\}$, look at the last equality in this sequence:

$$
m_{Y}(S) \mu_{S}+d_{S}=m_{Y, F}(S)+m_{1, S}+\ldots+m_{S-1, S}
$$

If $J_{m}^{+} \neq \emptyset$, then, by the part (ii) of Lemma 2.2, we obtain

$$
d_{S} \geq \sum_{i \in J_{m}^{+}} m_{Y}(i) \mu_{i} \operatorname{deg}\left(\varphi_{i-1, S}\right)_{*} B_{i-1} \geq \sum_{i \in J_{m}^{+}} m_{Y}(i) \mu_{i} .
$$

Recall the following useful

Definition 2.1. (see $[8,13]$ ). A function $a: J_{s} \rightarrow \mathbb{R}_{+}$is said to be compatible with the graph structure, if

$$
a(i) \geq \sum_{\substack{j \rightarrow i, j \in J_{s}}} a(j)
$$

for any $i \in J_{s}$.

We will actually use only one function, compatible with the graph structure, namely $a(i)=p_{i}$.

Proposition 2.2. Let $a(\cdot)$ be a function, compatible with the graph structure. Then the following inequality holds:

$$
\sum_{i \in J_{s}} a(i) m_{Y, F}(i) \geq \sum_{i \in J_{s}} a(i) m_{Y}(i) \mu_{i}+a(S) \sum_{i \in J_{m}^{+}} m_{Y}(i) \mu_{i} .
$$

Proof is obtained in exactly the same way as in the case of two divisors $([8,13])$ multiply the $i$-th equality by $a(i)$ put them all together. In the right hand side for any $i \geq 1$ we obtain the expression

$$
\sum_{j \geq i+1} a(j) m_{i, j}
$$

In the left hand side for any $i \geq 1$ we obtain the component $a(i) d_{i}$.

Lemma 2.3. If $m_{i, j}>0$, then $j \rightarrow i$.

Proof [8,13]: if $m_{i, j}>0$, then $B_{j-1} \subset \operatorname{Supp} Z_{i}^{j-1}$, but $\operatorname{Supp} Z_{i} \subset E_{i}$ so that $B_{j-1} \subset E_{i}^{j-1}$. Q.E.D. for the lemma.

The next standard step is to compare the multiplicities $m_{i, j}$ with the degrees. 
Lemma 2.4. For any $i<j \in J_{s}$ we get

$$
m_{i, j} \leq d_{i} .
$$

Proof. If $m_{i, j}=0$, then there is nothing to prove. Otherwise $j \rightarrow i$ and we need to prove that

$$
\operatorname{mult}_{B_{j-1}} Z_{i}^{j-1} \leq \operatorname{deg} Z_{i} .
$$

Taking into account that the maps $\varphi_{a, b}: B_{a} \rightarrow B_{b}$ are surjective, it suffices to prove the inequality

$$
\operatorname{mult}_{\left[B_{j-1} \cap \varphi_{i, i-1}^{-1}(t)^{j-1}\right]}\left[Z_{i} \cap \varphi_{i, i-1}^{-1}(t)\right]^{j-1} \leq \operatorname{deg}\left[Z_{i} \cap \varphi_{i, i-1}^{-1}(t)\right],
$$

where $t \in B_{i-1}$ is a point of general position. Taking into account that $\varphi_{i, i-1}^{-1}(t)$ is the projective space $\mathbb{P}^{\text {codim } B_{i-1}-1}$, we see that the right hand side in (20) means the usual degree of a hypersurface in the projective space whereas the set $\left[Z_{i} \cap\right.$ $\left.\varphi_{i, i-1}^{-1}(t)\right]^{j-1}$ is obtained from this hypersurface by means of a finite sequence of blow ups $\varphi_{s, s-1}, s=i+1, \ldots, j-1$, restricted onto $\varphi_{i, i-1}^{-1}(t)$. Taking into consideration that the multiplicities do not increase when blowing ups are performed, we reduce the claim to the obvious case of a hypersurface in the projective space. Q.E.D. for the lemma.

As a result, we obtain the following estimate

$$
\sum_{j \geq i+1} a(j) m_{i, j}=\sum_{\substack{j \geq i+1 \\ m_{i, j} \neq 0}} a(j) m_{i, j} \leq d_{i} \sum_{j \rightarrow i} a(j) \leq a(i) d_{i} .
$$

By what was said above, we may remove from the right hand side all the components $m_{i, *}, i \geq 1$ and from the left hand side all the components $d_{i}, i \geq 1$, replacing the equality sign $=$ by the inequality sign $\leq$. Q.E.D.

Setting in the inequality (19) $a(i)=p_{i}$ and recalling that for $j \geq S$ we have $p_{j} \leq p_{S}$, we complete the proof of Lemma 2.1.

Now let us complete the proof of Proposition 2.1.

It is obvious that the inequality (18) remains valid if $Y$ is an effective horizontal cycle of codimension 2 on $V$, that is, each component of the cycle $Y$ is a horizontal subvariety. Besides, the formulae (16), (17) extend by linearity to the set of all effective horizontal cycles, and the left hand and the right hand sides of the inequality (18) are linear in $m_{Y}(\cdot), m_{Y, F}(\cdot)$, respectively.

Now set $Y=Z^{h}$ and take into account that

$$
m_{Y, F}(i) \leq \operatorname{mult}_{B_{1}}\left(Z^{h} \circ F\right)^{1}
$$

for $i \geq 2$. This implies the inequality (14). The second inequality of Proposition 2.1 follows from (14). Q.E.D. for the proposition.

Remark 2.1. The inequality (15) is more compact than (14), however in some cases it is possible to get a stronger estimate for mult $_{B_{1}}\left(Z^{h} \circ F\right)^{1}$ than for mult $_{B}\left(Z^{h} \circ\right.$ $F)$. 


\subsection{Estimating the multiplicities of a linear system: the non-singular case}

Now let us turn to the main problem, that is, estimating the singularities of the movable linear system $\Sigma$. Assume that the fiber space $V / \mathbb{P}^{1}$ satisfies the conditions (v) and (vs), and at any point $o \in V$ satisfies at least one of the conditions (f) or (fs) formulated below:

(f) for any irreducible subvariety $Y \ni o \in F$ of codimension 2 (with respect to the fiber $F$ ) the following inequality holds:

$$
\frac{\text { mult }_{o}}{\operatorname{deg}} Y \leq \frac{4}{\operatorname{deg} V},
$$

(fs) $o \in F$ is a double point of the fiber and for any irreducible subvariety $Y \subset F$ of codimension 2 the following estimate holds:

$$
\frac{\text { mult }_{o}}{\operatorname{deg}} Y \leq \frac{6}{\operatorname{deg} V},
$$

and, besides, for any infinitely near point of the first order $x \in E_{F}$, where $\varphi_{o}: \widetilde{F} \rightarrow F$ is the blow up of the point $o \in F, E_{F} \subset \widetilde{F}$ the exceptional divisor, the inequality

$$
\frac{\operatorname{mult}_{x} \tilde{Y}}{\operatorname{deg} Y} \leq \frac{3}{\operatorname{deg} V}
$$

holds, where $\widetilde{Y}$ is the strict transform of the subvariety $Y$ on $\widetilde{F}$.

Remark 2.2. Let us draw the reader's attention to the fact that in the condition (f) we do not specify whether the point $o \in F$ is singular or smooth. For the varieties of general position, considered in this paper, all smooth points satisfy the condition (f) whereas the singular points are of different behavior. This point is discussed below in $\S 3$.

Assume first that $B=\operatorname{centre}\left(\nu_{E}, V\right) \not \subset \operatorname{Sing} F$. In other words, either the fiber $F$ is non-singular or $B$ is not a singular point of this fiber. By the regularity condition, for any irreducible subvariety $Y \subset F$ of codimension two (with respect to $F$ ) the estimate (21) holds for a point $o \in B$ of general position. For all the multiplicities we have $\mu_{i}=1$. The inequality (21) implies immediately the estimate

$$
\sum_{i \in J_{s} \cup J_{m}^{+}} p_{i} m_{i}^{h} \leq 4 n^{2} \Sigma_{s} .
$$

Since $m_{i}^{h} \leq m_{1}^{h} \leq 4 n^{2}$, we get the inequality

$$
\sum_{i \in J_{l}} p_{i} m_{i}^{h} \leq 4 n^{2}\left(\Sigma_{s}+\Sigma_{m}^{-}\right) .
$$

This is the required estimate of singularities of the horizontal component $Z^{h}$. Consider the vertical component $Z^{v}$. By the condition (v) the inequality

$$
m_{i}^{v} \leq m_{1}^{v} \leq \frac{2}{\operatorname{deg} V} d_{v} .
$$


holds. On the other hand, the generalized $K^{2}$-condition of depth $\varepsilon$ implies the estimate

$$
\frac{d_{v}}{\operatorname{deg} V}<\frac{2 e n}{\nu_{E}(F)}+\varepsilon n^{2} .
$$

Combining (24) and (25), we obtain the inequality

$$
\sum_{i \in J_{s} \cup J_{m}^{+}} p_{i} m_{i}^{v}<2 n\left(\frac{2 e}{\nu_{E}(F)}+\varepsilon n\right)\left(\Sigma_{s}+\Sigma_{m}^{+}\right) .
$$

Taking into account that by definition $\nu_{E}(F)=\sum_{i=1}^{k} p_{i} \mu_{i} \geq \Sigma_{s}+\Sigma_{m}^{+}$, we obtain finally

$$
\sum_{i \in J_{s} \cup J_{m}^{+}} p_{i} m_{i}^{v}<4 n e+2 \varepsilon n^{2}\left(\Sigma_{s}+\Sigma_{m}^{+}\right)
$$

Now the inequalities (66), (223) and (26) lead to the following estimate:

$$
\begin{aligned}
\left(4 n^{2}\left(\Sigma_{s}+\Sigma_{m}^{-}\right)\right. & \left.+4 n e+2 \varepsilon n^{2}\left(\Sigma_{s}+\Sigma_{m}^{+}\right)\right)\left(\Sigma_{s}+\Sigma_{m}+\Sigma_{u}\right)> \\
> & \left(\left(3 \Sigma_{s}+2 \Sigma_{m}+\Sigma_{u}\right) n+e\right)^{2} .
\end{aligned}
$$

Setting in this inequality $\varepsilon=2$ and taking into account that $\Sigma_{m}=\Sigma_{m}^{+}+\Sigma_{m}^{-}$(this is the crucial point), after some easy arithmetic we get the inequality

$$
\left(n\left(\Sigma_{s}-\Sigma_{u}\right)+e\right)^{2}<0 .
$$

A contradiction.

The equality of the thresholds of canonical adjunction

$$
c_{\mathrm{virt}}(\Sigma)=c(\Sigma)
$$

is proved for the non-singular case $B \not \subset$ Sing $F$.

\subsection{Estimating the multiplicities of a linear system: the singular case}

Now consider the case when $B=o \in F$ is a singular point of the fiber. If the variety $F$ satisfies the condition (f) at the point $o$, then the arguments of the previous section work well without any modifications. If this is not the case, then $\operatorname{deg} V=\operatorname{deg} F \geq 6$ (otherwise $\operatorname{deg} V \leq 4$ and the condition (f) holds automatically) and thus $\operatorname{dim} F \geq 4$, $\operatorname{dim} V \geq 5$. Therefore the discrepancy of the first exceptional divisor $E_{1}$ is at least 4 (we blow up a smooth point $o \in V$ ). By assumption, the condition (fs) holds, whence we get the inequality

$$
m_{i}^{h} \leq m_{1}^{h} \leq 3 n^{2}
$$


so that from the estimate (14) we obtain

$$
\sum_{i \in J_{l}} p_{i} m_{i}^{h} \leq 3 n^{2}\left(p_{1}+\Sigma_{s}+\Sigma_{m}^{-}\right)
$$

On the other hand, as we have mentioned above, $a\left(E_{1}, V\right) \geq 4$, so that one can replace in the inequality (6) the numerator by $\left(p_{1}+3 \Sigma_{s}+2 \Sigma_{m}+\Sigma_{u}\right)$. Now arguing as in Sec. 2.4 we get the inequality

$$
\begin{gathered}
\left(n\left(\Sigma_{s}-\Sigma_{u}\right)+e\right)^{2}+\left(\Sigma_{s}-3 p_{1}\right)\left(\Sigma_{s}+\Sigma_{m}+\Sigma_{u}\right) n^{2}+ \\
\quad+n p_{1}\left(n p_{1}+2\left(3 \Sigma_{s}+2 \Sigma_{m}+\Sigma_{u}\right) n+2 e\right)<0 .
\end{gathered}
$$

Obviously, $\Sigma_{s}-3 p_{1} \geq-2 p_{1}$ so that we again get a contradiction.

The proof of the equality of the thresholds of canonical adjunction $c_{\mathrm{virt}}(\Sigma)=c(\Sigma)$ is complete.

Because of the importance of this result for further work let us formulate it as a separate claim.

Theorem 2. Assume that the fiber space $V / \mathbb{P}^{1}$ satisfies the generalized $K^{2}$ condition of depth 2, the conditions (v), (vs) and at least one of the conditions ( $f$ ) or (fs) at every point $o \in V$. Then for any movable linear system $\Sigma \subset\left|-n K_{V}+l F\right|$ with $l \in \mathbb{Z}_{+}$its virtual and actual thresholds of canonical adjunction coincide:

$$
c_{\mathrm{virt}}(\Sigma)=c(\Sigma)
$$

\section{Varieties with a pencil of Fano double covers}

In order to apply the technique developed above to the fiber spaces $V / \mathbb{P}^{1}$, the fibers of which are Fano double hypersurfaces, one needs to describe movable linear systems on these varieties and check the conditions on multiplicities of horizontal and vertical cycles for all the families under consideration: $1-8$ and $1^{*}-5^{*}$ of Sec. 0.2 and 0.3 .

\subsection{Movable systems on the varieties of the type $((0),(2,0))$}

Let $V / \mathbb{P}^{1}$ be a variety from the family $((0),(2,0))$. The construction of the variety $V$ can be alternatively described in the following way.

Let $W_{\mathbb{P}} \subset \mathbb{P}$ be a hypersurface of degree $2 l$,

$$
\sigma_{\mathbb{Y}}: \mathbb{Y} \rightarrow \mathbb{P}
$$

the double cover branched over the divisor $W_{\mathbb{P}}$. Consider the variety $Y=\mathbb{P}^{1} \times \mathbb{Y}$, which is realized as the double cover $\sigma_{Y}: Y \rightarrow X=\mathbb{P}^{1} \times \mathbb{P}$ branched over the divisor $W=\mathbb{P}^{1} \times W_{\mathbb{P}}$. Set $V=\sigma_{Y}^{-1}(Q)$, where $Q \subset X$ is a smooth divisor of the type $(2, m)$. It is easy to see that in this way we obtain the same variety $V$ as in Sec. 0.2. 
By assumption, the divisor $Q \subset \mathbb{P}^{1} \times \mathbb{P}$ is given by the equation

$$
A\left(x_{*}\right) u^{2}+2 B\left(x_{*}\right) u v+C\left(x_{*}\right) v^{2}=0,
$$

where $A(\cdot), B(\cdot), C(\cdot)$ are homogeneous of degree $m$. Here $(u: v)$ and $\left(x_{*}\right)=$ $\left(x_{0}: \ldots: x_{M+1}\right)$ are homogeneous coordinates on $\mathbb{P}^{1}$ and $\mathbb{P}$, respectively.

Furthermore, let $H_{\mathbb{P}}$ be the class of a hyperplane in $\mathbb{P}, L_{X}=p_{X}^{*} H_{\mathbb{P}}$ the tautological class on $X$, where $p_{X}: X \rightarrow \mathbb{P}$ is the projection onto the second factor, $L_{V}=\left.\sigma_{Y}^{*} L_{X}\right|_{V}$. It is easy to see that

$$
K_{V}=-L_{V}
$$

so that the anticanonical linear system $\left|-K_{V}\right|$ is free and determines the projection $p_{V}=p_{X} \circ \sigma: V \rightarrow \mathbb{P}$.

Lemma 3.1. The projection $p_{V}$ factors through the double cover $\sigma_{\mathbb{Y}}: \mathbb{Y} \rightarrow \mathbb{P}$. More precisely, there is a morphism $p: V \rightarrow \mathbb{Y}$ such that

$$
p_{V}=\sigma_{\mathbb{Y}} \circ p .
$$

The degree of the morphism $p$ at a general point is equal to 2.

Proof. Consider a point $x \in \mathbb{P} \backslash W_{\mathbb{P}}$ of general position. Set $\left\{y^{+}, y^{-}\right\}=\sigma_{\mathbb{Y}}^{-1}(x) \subset$ $\mathbb{Y}$. Set also

$$
L_{x}=\mathbb{P}^{1} \times\{x\} \subset X, \quad L_{x}^{ \pm}=\mathbb{P}^{1} \times\left\{y^{ \pm}\right\} \subset Y .
$$

It is obvious that the inverse image $\sigma_{Y}^{-1}\left(L_{x}\right)$ is the disjoint union of the lines $L_{x}^{+}$and $L_{x}^{-}$, whereas

$$
p_{Y}\left(L_{x}^{ \pm}\right)=y^{ \pm},
$$

where $p_{Y}: Y \rightarrow \mathbb{Y}$ is the projection onto the second factor. The divisor $Q$ intersects $L_{x}$ at two distinct (for a general point $x$ ) points $q_{1}, q_{2}$. Set

$$
\sigma^{-1}\left(q_{i}\right)=\left\{o_{i}^{+}, o_{i}^{-}\right\} \subset V, \quad o_{i}^{ \pm} \in L_{x}^{ \pm} .
$$

The morphism $p$ is the restriction $\left.p_{Y}\right|_{V}$. Obviously,

$$
p^{-1}\left(y^{ \pm}\right)=\left\{o_{1}^{ \pm}, o_{2}^{ \pm}\right\},
$$

where the sign + or - is the same in the right hand and left hand side. This proves the lemma.

Let $\Delta \subset V$ be a subvariety of codimension 2, given by the system of equations $A=B=C=0$. The subvariety $\Delta$ is swept out by the lines $L_{y}=\mathbb{P}^{1} \times\{y\}$ which are contracted by the morphism $p$. Set $\Delta_{\mathbb{Y}}=p(\Delta)$. Obviously,

$$
p: V \backslash \Delta \rightarrow \mathbb{Y} \backslash \Delta_{\mathbb{Y}}
$$

is a finite morphism of degree 2. Let $\tau \in \operatorname{Bir} V$ be the corresponding Galois involution. It is easy to see that $\tau$ commutes with the Galois involution $\alpha \in$ Aut $V$ of the double cover $\sigma: V \rightarrow Q$, so that $\tau$ and $\alpha$ generate a group of four elements. Since 
the involution $\tau$ is biregular outside the invariant closed subset $\Delta$ of codimension 2 , that is, $\tau \in \operatorname{Aut}(V \backslash \Delta)$, the action of $\tau$ on the Picard group Pic $V$ is well defined.

Let $\Sigma \subset\left|-n K_{V}+l F\right|$ be a movable linear system.

Lemma 3.2. (i) The involution $\tau$ transforms the pencil $|F|$ of fibers of the morphism $\pi$ into the pencil $\left|m L_{V}-F\right|$. (ii) If $l<0$, then the involution $\tau$ transforms the linear system $\Sigma$ into the linear system

$$
\Sigma^{+} \subset\left|n^{+} L_{V}+l^{+} F\right|,
$$

where $n^{+}=n+l m \geq 0, l^{+}=-l>0$.

Proof. Obviously, $\tau^{*} L_{V}=L_{V}$. Let $F_{t}=\pi^{-1}(t)$ be a fiber. We get

$$
p^{-1}\left(p\left(F_{t}\right)\right)=F_{t} \cup \tau\left(F_{t}\right) .
$$

However, $p\left(F_{t}\right) \sim m H_{\mathbb{Y}}=m \sigma_{\mathbb{Y}}^{*} H_{\mathbb{P}}$ by the construction of the variety $V$. Since $p^{*} H_{\mathbb{Y}}=L_{V}$, we obtain the claim (i). Thus $\tau^{*} F=m L_{V}-F$. This directly implies the second claim of the lemma.

Remark 3.1. Varieties of the type $((0),(2,0))$ are similar by their properties to the Fano fiber spaces considered in [19].

\subsection{Checking the $K$-condition}

Let us prove that the variety $V / \mathbb{P}^{1}$ from any of the families $2-8$ or $1^{*}-5^{*}$ of Sec. $0.2,0.3$ satisfies the $K$-condition. Fix a movable linear system $\Sigma \subset\left|-n K_{V}+l F\right|$. We must show that $l \in \mathbb{Z}_{+}$. We will use the arguments of the following two types: (1) assume that there is a divisor $E \subset V$, swept out by a family of irreducible horizontal curves $\left(C_{\delta}, \delta \in \Delta\right)$ such that

$$
\left(-K_{V} \cdot C_{\delta}\right) \leq 0
$$

Since the linear system $\Sigma$ is movable we get $l \geq 0$, which is what we need.

(2) Assume that there is a horizontal prime divisor $E$ on $V$ satisfying the inequality

$$
\left(-K_{V} \cdot E \cdot L_{V}^{M-1}\right) \leq 0 .
$$

For a general divisor $D \in \Sigma$ the cycle $(D \circ E)$ is effective and thus

$$
\left(D \cdot E \cdot L_{V}^{M-1}\right) \geq 0
$$

whence, taking into account that $\left(H_{F} \cdot L_{V}^{M-1}\right)>0$, we get $l \geq 0$.

Now let us consider varieties of the types $2-8$ and $1^{*}-5^{*}$ one after another.

\subsubsection{Varieties of the type $((0),(1,1))$}

Here $X=\mathbb{P}^{1} \times \mathbb{P}, Q \subset X$ is given by the equation $A u+B v=0$ where $A, B$ are homogeneous polynomials of degree $m$ on $\mathbb{P}$. The pair of equations $A=B=0$ defines on $Q$ the divisor $\Delta=\mathbb{P}^{1} \times \Delta_{\mathbb{P}}$, which is swept out by horizontal lines, 
$\Delta_{\mathbb{P}} \subset \mathbb{P}$ is the subvariety $\{A=B=0\}$ of codimension two. For any line $L_{x}=$ $\sigma^{-1}\left(\mathbb{P}^{1} \times\{x\}\right), x \in \Delta_{\mathbb{P}}$, we get

$$
\left(-K_{V} \cdot L_{x}\right)=\left(L_{V} \cdot L_{x}\right)=0 .
$$

Now the remark (1) shows that the $K$-condition holds.

\subsubsection{Varieties of the type $((1),(0,1))$}

Here

$$
\mathcal{E}=\mathcal{O}_{\mathbb{P}^{1}}^{\oplus(M+1)} \oplus \mathcal{O}_{\mathbb{P}^{1}}(1),
$$

and set $E \subset X$ to be the divisor of common zeros of all sections $s \in \mathcal{O}_{\mathbb{P}^{1}}(1)$. Obviously, $E=\mathbb{P}^{1} \times \mathbb{P}^{M}$. Let $L_{E}=p_{E}^{*}\left(\left.H_{\mathbb{P}}\right|_{\mathbb{P} M}\right)$ be the tautological class on $E$, where $p_{E}: E \rightarrow \mathbb{P}^{M}$ is the projection onto the second factor. We get

$$
-\left.K_{V}\right|_{E}=\left.L_{V}\right|_{E}=L_{E},
$$

$E \cap Q \sim m L_{E}$, so that $E \cap Q=\mathbb{P}^{1} \times Q_{E}$, where $Q_{E} \subset \mathbb{P}^{M}$ is a hypersurface of degree $m$. Therefore, $V$ contains the divisor $\sigma^{-1}(E \cap Q)$, which is swept out by the curves $L_{x}=\sigma^{-1}(\mathbb{P} \times\{x\}), x \in Q_{E}$. The anticanonical class $\left(-K_{V}\right)$ is trivial on these curves. According to the remark (1), the $K$-condition holds.

\subsubsection{Varieties of type $((2),(1,0))$}

Here $-K_{V}=L_{V}-F$

$$
\mathcal{E}=\mathcal{O}_{\mathbb{P}^{1}}^{\oplus(M+1)} \oplus \mathcal{O}_{\mathbb{P}^{1}}(2),
$$

set $E \subset X$ to be the divisor of common zeros of sections $s \in \mathcal{O}_{\mathbb{P}^{1}}(2)$. Now

$$
\sigma^{-1}(E \cap Q) \in\left|L_{V}-2 F\right| .
$$

It is easy to compute that

$$
\left(-K_{V} \cdot\left(L_{V}-2 F\right) \cdot L_{V}^{M-1}\right)=2 n(1-m) \leq 0 .
$$

According to the remark (2), the $K$-condition holds.

\subsubsection{Varieties of the type $((2),(0,0))$}

Here $-K_{V}=L_{V}$ and we can apply the remark (1): the divisor $\sigma^{-1}(E \cap Q) \subset V$ (in the notations of the previous case) is swept out by horizontal curves. The class $L_{V}$ is trivial on these curves.

\subsubsection{Varieties of the type $((3),(0,0))$}

Here

$$
\mathcal{E}=\mathcal{O}_{\mathbb{P}^{1}}^{\oplus(M+1)} \oplus \mathcal{O}_{\mathbb{P}^{1}}(3)
$$

and $-K_{V}=L_{V}-F$. Set $E \subset X$ to be the divisor of common zeros of the sections $s \in \mathcal{O}_{\mathbb{P}^{1}}(3)$. Now the divisor $\sigma^{-1}(E \cap Q) \subset V$ is swept out by horizontal curves, on which $L_{V}$ is trivial. We apply the remark (1). 


\subsubsection{Varieties of the type $((1,2),(0,0))$}

Here $-K_{V}=L_{V}-F$ and there is a prime divisor $E \subset V$, such that

$$
E \sim L_{V}-2 F .
$$

It is easy to check that

$$
\left(\left(L_{V}-2 F\right) \cdot\left(L_{V}-F\right) \cdot L_{V}^{M-1}\right)=0 .
$$

By the remark (2), the $K$-condition holds.

\subsubsection{Varieties of the type $((1,1,1),(0,0))$}

Here $-K_{V}=L_{V}-F$ and moreover $\operatorname{codim}_{V} \mathrm{Bs}\left|L_{V}-F\right|=3$. Thus for any pseudoeffective class $D \in \operatorname{Pic} V$ we get the inequality

$$
\left(D \cdot\left(L_{V}-F\right)^{2} \cdot L_{V}^{M-2}\right) \geq 0 .
$$

However, it is easy to compute that $\left(\left(L_{V}-F\right)^{3} \cdot L_{V}^{M-2}\right)=0$. Thus if $D=-n K_{V}+l F$ is just a pseudo-effective class, then $l \in \mathbb{Z}_{+}$. In particular, the $K$-condition holds.

The remaining five types of double spaces are considered in the same way (with simplifications).

Q.E.D. for the $K$-condition for varieties of types $2-8$ and $1^{*}-5^{*}$.

\subsection{Proof of birational rigidity}

Now in order to prove Theorem 1 , we have to check the equality

$$
c_{\mathrm{virt}}(\Sigma)=c(\Sigma)
$$

for any movable linear system $\Sigma \subset\left|-n K_{V}+l F\right|$ with $n \geq 1$ and $l \in \mathbb{Z}_{+}$. In its turn, by Theorem 2 it is sufficient to verify that varieties of the types $1-8$ and $1^{*}-5^{*}$ satisfy the generalized $K^{2}$-condition of depth 2 and that a regular fiber space $V / \mathbb{P}^{1}$ satisfies the conditions (v), (vs) and (f) or (fs) at every point (the condition (h) follows directly from any of the two conditions (f) or (fs)). The conditions (v) and (vs) are checked in [16], the condition (f) for a regular smooth point of a fiber $o \in F$ is checked in [9]. It remains to show that at a double point $o$ the fiber $F$ satisfies at least one of the two conditions (f) or (fs).

If the singular point $o \in F$ lies outside the branch divisor of the morphism $\sigma$, then the condition (f) is satisfied. This is easy to check by means of the standard method of hypertangent divisors. One should take into account that if $o \in F$ does not lie on the branch divisor, then there are $l$ additional hypertangent divisors, arising from the double cover. It is this fact that makes it possible to obtain the estimate (21) for any irreducible subvariety $Y \subset F$ of codimension 2 (with respect to the fiber $F$ ). We do not give these arguments here because they are standard 
and, in particular, parallel to another estimate which is proved below for smooth points (where 4 is replaced by 3 ).

Therefore let us assume that the singular point $o \in F$ lies on the branch divisor. Let $Y \subset F$ be an irreducible subvariety of codimension $2, T=\sigma^{-1}\left(T_{p} G \cap G\right)$, $p=\sigma(o)$, the tangent divisor. If $Y \not \subset T$, then considering the effective cycle $(Y \circ T)$ of codimension 3 and applying the standard technique of hypertangent divisors to this cycle (that is, intersecting it with $D_{i} \in \Lambda_{i}, i=4, \ldots, m-1$ ), we obtain the inequality (21). Thus we may assume that $Y \subset T$. Applying the technique of hypertangent divisors (with $D_{i} \in \Lambda_{i}$ for $i=3, \ldots, m-1$ ) we obtain (22), the first of the two inequalities of the condition (fs). Let us prove the second one.

Let $\varphi: \widetilde{T} \rightarrow T$ be the blow up of the point $o, E=\varphi^{-1}(o) \subset \widetilde{T}$ the exceptional divisor. It is easy to see that the double cover $\sigma$ presents $E$ as the double cover of the quadric $E_{G}$ branched over the divisor $\widetilde{W} \cap E_{G}$, that is, the section of $E_{G}$ by a quadric hypersurface. The quadric $E_{G}$ is the exceptional divisor of the blow up of the point $p=\sigma(o)$ on the variety $T_{p} G \cap G, \widetilde{W}$ denotes the strict transform of the restriction $\left.W\right|_{G}$. By assumption, $Y$ is a prime divisor on $T$. Therefore, $Y_{E}=(\tilde{Y} \circ E)$ is an effective divisor on the double quadric $E$, and $\operatorname{deg} Y_{E}=\operatorname{mult}_{o} Y$. Let $x \in E$ be an arbitrary point.

Set $q=\sigma(x) \in E_{G}, R=\sigma^{-1}\left(T_{q} E_{G} \cap E_{G}\right)$. The divisor $R \subset E$ is irreducible, $\operatorname{deg} R=4$, mult $_{x} R=2$. If $Z \subset E$ is a prime divisor, different from $R$, then the cycle $(Z \circ R)$ of codimension 2 on $E$ is well defined, and moreover

$$
\operatorname{deg} Z=\operatorname{deg}(Z \circ R) \geq \operatorname{mult}_{x}(Z \circ R) \geq 2 \operatorname{mult}_{x} Z .
$$

This implies that

$$
\operatorname{mult}_{x} \tilde{Y} \leq \operatorname{mult}_{x} Y_{E} \leq \frac{1}{2} \operatorname{deg} Y_{E}=\frac{1}{2} \operatorname{mult}_{o} Y .
$$

This proves the second inequality of the condition (fs). As for the generalized $K^{2}$ condition of depth 2 , it follows from the inequality

$$
\left(\left(K_{V}^{2}-2 H_{F}\right) \cdot L_{V}^{M-1}\right) \leq 0
$$

which is easy to check for varieties of types $1-8$ and $1^{*}-5^{*}$ of Sec. 0.2 and 0.3 .

Q.E.D. for Theorem 1.

\subsection{Multiplicities of subvarieties of codimension 2}

In the remaining part of this section we prove the following claim.

Proposition 3.1. Let $o \in F$ be a smooth point of the fiber lying outside the branch divisor of the morphism $\sigma$. Then for any irreducible subvariety $Y \subset F$ of codimension two (and thus for any effective cycle of pure codimension two) the following estimate is true:

$$
\frac{\text { mult }_{o}}{\operatorname{deg}} Y \leq \frac{3}{\operatorname{deg} V}=\frac{3}{2 m}
$$


Remark 3.2. Proposition 3.1 outlines another approach to proving birational rigidity of the varieties under consideration, based on Proposition 1.7. If a smooth point $o \in F$ lies on the branch divisor of the morphism $\sigma$, then there exists an irreducible subvariety $Y^{+} \subset F$ of codimension 2 for which the estimate of the condition (f) cannot be improved: the linear system $|H-2 o|$ is movable in this case and spanned by two divisors, $\sigma^{*}\left(T_{p} G \cap G\right)$ and $\sigma^{*}\left(T_{p} W \cap G\right)$, where $p=\sigma(o)$. The intersection of these divisors gives a subvariety $Y^{+}$with this property. It can be shown, however, that for any other subvariety of codimension two, $Y \neq Y^{+}$the inequality (27) holds. As for the subvariety $Y^{+}$, it satisfies the estimate (27) at all other points, including the infinitely near points of the first order lying over the point $o$. Now using the discrepancy arguments one can prove birational rigidity using only Proposition 1.7. However, a combination of these ideas with Theorem 2 makes it possible to improve the technique even further. We will use this strategy in the subsequent papers.

Proof of Proposition 3.1. Arguing in the same way as in [16], $\S 2$, we give two methods of obtaining the estimate (27). The first one is much more simple, however works only for sufficiently high dimensions $M \geq M_{0}$. The second method gives the estimate (27) for all dimensions, however it requires additional conditions of general position which one has to substantiate (Sec. 3.5). Whichever method is used, the first step is the same.

Lemma 3.3. Assume that the inequality

$$
\frac{\text { mult }_{o}}{\operatorname{deg}} Y>\frac{3}{2 m}
$$

holds. Then $\sigma(Y) \subset T_{p} G$, where $p=\sigma(o)$.

Proof. Assume the converse: $\sigma(Y) \not \subset T_{p} G$. Then the intersection $Y \cap T$, where $T=\sigma^{*}\left(T_{p} G \cap G\right)$ is the tangent section of the fiber $F$ at the point $o$, is of codimension 3 and therefore the effective cycle $(Y \circ T)$ is well defined. For some component $Y_{3}$ of this cycle the inequality

$$
\frac{\text { mult }_{o}}{\operatorname{deg}} Y_{3}>\frac{3}{m}
$$

holds. Now arguing in the usual way let us consider general divisors of the hypertangent linear systems

$$
D_{i} \in \Lambda_{i}, \quad D_{j}^{+} \in \Lambda_{j}
$$

where

$$
i \in\{4, \ldots, m-1\}, \quad j \in\{l, \ldots, 2 l-2\}
$$

for $m \leq 2 l$ and

$$
i \in\{4, \ldots, m-2\}, \quad j \in\{l, \ldots, 2 l-1\}
$$

for $m \geq 2 l+1$. Taking into account the codimension of the base set of the system $\Lambda_{i}$, we see that the set

$$
Y_{3} \cap\left(\bigcap_{i} D_{i}\right) \cap\left(\bigcap_{j} D_{j}^{+}\right)
$$


is one-dimensional in a neighborhood of the point $o$. From this we obtain in the standard way the estimate

$$
\frac{\text { mult }_{o}}{\operatorname{deg}} Y_{3} \leq \frac{4 l}{m(2 l-1)}
$$

for $m \leq 2 l$ and the estimate

$$
\frac{\text { mult }_{o}}{\operatorname{deg}} Y_{3} \leq \frac{2}{m-1}
$$

for $m \geq 2 l+1$. In any case the estimates (30) and (31) are incompatible with the inequality (29). Q.E.D. for the lemma.

Now let us prove Proposition 3.1. If the estimate (27) does not hold, that is, if the estimate (28) holds, then by what was proved above, $\sigma(Y) \subset T_{p} G$, that is,

$$
Y \subset T_{1}=\sigma^{-1}\left(G \cap T_{p} G\right)
$$

By the regularity conditions, $T_{1} \subset F$ is an irreducible subvariety with the isolated double point $o \in T_{1}$. Consider the closed set

$$
T_{12}=\sigma^{-1}\left(\sigma\left(T_{1}\right) \cap T_{p} \sigma\left(T_{1}\right)\right)
$$

By the regularity conditions, $T_{12} \subset F$ is an irreducible subvariety of codimension two, and moreover mult ${ }_{o} T_{12}=6$, so that the equality

$$
\frac{\text { mult }_{o}}{\operatorname{deg}} T_{12}=\frac{3}{2 m}
$$

holds, whence by the inequality (28) we get, that $Y \neq T_{12}$. As above, let

$$
D_{i} \in \Lambda_{i}, D_{j}^{+} \in \Lambda_{j}^{+}
$$

be general hypertangent divisors, where

$$
i \in I=\{2,4, \ldots, m-1\}, \quad j \in J=\{l, \ldots, 2 l-2\}
$$

(in $I$ we omit the element $i=3$ ) for $m \leq 2 l$ and

$$
i \in I\{2,4, \ldots, m-2\}, \quad j \in J=\{l, \ldots, 2 l-1\}
$$

(in $I$ we again omit $i=3$ ) for $m \geq 2 l+1$. By the regularity conditions the intersection

$$
Y \cap\left(\bigcap_{i \in I} D_{i}\right) \cap\left(\bigcap_{j \in J} D_{j}^{+}\right)
$$

is one-dimensional in a neighborhood of the point $o$, whence we get the estimates

$$
\frac{\text { mult }_{o}}{\operatorname{deg}} Y \leq \frac{8 l}{3 m(2 l-1)}
$$


and

$$
\frac{\text { mult }_{o}}{\operatorname{deg}} Y \leq \frac{4}{3(m-1)}
$$

for $m \leq 2 l$ and $m \geq 2 l+1$, respectively. It is easy to check that these estimates prove Proposition 3.1 in all cases except for the following ones:

$$
m \in\{3,4,5,6,7,8,\}, \quad l \in\{3,4\} .
$$

Now let us give a more refined way of arguing, which requires strong regularity conditions but works in all dimensions including low ones. This method is completely similar to the method by means of which the condition (vs) was proved in [16] for a double point of the fiber $o \in F$ outside the branch divisor. For this reason here we just describe the main steps of the proof, emphasizing the changes which should be made in the arguments of the paper [16].

So let us assume that for an irreducible variety $Y \subset F$ the inequality (28) holds, which contradicts the required estimate (27). By Lemma 3.3, $Y \subset T_{1}$. The point $o$ is an isolated factorial singularity of the variety $T_{1}$. Moreover, $\operatorname{Pic} T_{1}=\mathbb{Z} H_{T}$, where

$$
H_{T}=\left.H_{F}\right|_{T_{1}}
$$

is the class of a hyperplane section. Let $\varphi_{T}: \widetilde{T} \rightarrow T_{1}$ be the blow up of the point $o$, $E_{T} \subset \widetilde{T}$ the exceptional divisor (it is irreducible), $\widetilde{Y} \subset \widetilde{T}$ the strict transform of the divisor $Y$. We get

$$
\widetilde{Y} \sim \alpha H_{T}-\beta E_{T},
$$

where $\beta / \alpha>3 / 2$ by the estimate (28).

Lemma 3.4. The prime divisor $T_{12} \subset T_{1}$ is swept out by a family of curves $\left\{C_{\delta}, \delta \in \Delta\right\}$, the general curve of which is irreducible and satisfies the estimate

$$
\frac{\text { mult }_{o}}{\operatorname{deg}} C_{\delta}>\frac{2}{3} .
$$

Assume that the lemma is proved and consider the strict transform $\widetilde{C}_{\delta} \subset \widetilde{T}$ of the general curve $C_{\delta}, \delta \in \Delta$. Obviously,

$$
\left(\widetilde{Y} \cdot \widetilde{C}_{\delta}\right)<0,
$$

so that $C_{\delta} \subset Y$ and thus $T_{12} \subset Y$. Consequently, $Y=T_{12}$ which is impossible. Q.E.D. for Proposition 3.1.

To prove Lemma 3.4, one needs the arguments which are absolutely similar to the arguments that were used in the proof of Lemma 2.2 in [16], so that we will not repeat them, just remind the main steps. For $m \geq 4, l \geq 3$ define the sequence of integers

$$
c_{e}=\sharp[4, e] \cap \mathcal{M}+\sharp[3, e] \cap \mathcal{L}, \quad e \in \mathbb{Z}_{+},
$$

where $\mathcal{M}=\{2, \ldots, m-1\}, \mathcal{L}=\{l, \ldots, 2 l-1\}$, and construct the ordering function

$$
\chi:\{1, \ldots, m+l-4\} \rightarrow \mathbb{Z}_{+},
$$


setting $\chi\left(\left[c_{e-1}+1, c_{e}\right] \cap \mathbb{Z}_{+}\right)=e$. By the regularity condition, for a general set of hypertangent divisors

$$
\mathbb{D}=\left\{D_{i} \in \Lambda_{\chi(i)}^{P}, \quad i=1, \ldots, m+l-4\right\} \in \Lambda^{P}
$$

the closed algebraic set

$$
R_{i}(\mathbb{D})=\bigcap_{j=1}^{i} D_{i} \cap T_{P}
$$

is of codimension $i$ in $T_{12}$ for $i=1, \ldots, m+l-4$. Thus we get an effective 1 -cycle

$$
R(\mathbb{D})=R(\mathbb{D})=\left(T_{P} \circ D_{1} \circ \ldots \circ D_{m+l-4}\right)=\sum_{\delta_{i} \in \Delta} C_{\delta_{i}}+\Phi
$$

where $\left(C_{\delta}, \delta \in \Delta\right)$ is a movable family of curves, sweeping out $T_{12}, \Phi$ is the fixed part of the family $R(\mathbb{D})$. It is easy to see that $\Phi$ is exactly the 1 -cycle of lines on $F$, passing through the point $o \in F$, that is, $\operatorname{deg} \Phi=\operatorname{deg} \mathbb{L}(o)$. Therefore,

$$
\frac{\text { mult }_{o}}{\operatorname{deg}} C_{\delta} \geq \frac{\frac{m !}{4} \cdot \frac{(2 l) !}{l !}-\lambda_{m, l}}{\frac{2 m !}{3} \cdot \frac{(2 l-1) !}{(l-1) !}-\lambda_{m, l}}>\frac{2}{3}
$$

by Proposition 3.2 (which is proved below). In a similar way we argue when $m=3$ or $l=2$. Proof of Lemma 3.3 is complete.

Let us emphasize that in contrast to the situation considered in [16], here the hardest point is to estimate the number of lines (taken with multiplicities), passing through the point $o \in F$. In [16] the double points of the fibers are considered. There are but finitely many such points so that for a general Fano fiber space $V / \mathbb{P}^{1}$ all the lines passing through a singular point of a fiber are of multiplicity one, and this task becomes trivial. However, in out case $o \in F$ is an arbitrary point of a fiber. As we will show just now, the multiplicity of a line passing through some specially chosen point $o \in F$ can be very high. But not high enough to prevent the method of proving Lemma 2.2 in [16] to work in our case. All the other differences between the proof of Proposition 3.1 of this paper and that of Proposition 2.3 in [16] are inessential.

\subsection{Estimating the number of lines}

For a point $o \in V \backslash \sigma^{-1}(W)$ outside the branch divisor we define the algebraic cycle of lines $\mathbb{L}(o)$ on $V$ passing through $o$ as the 0 -cycle of the subscheme

$$
\left\{q_{1}=\ldots=q_{m}=g_{l+1}=\ldots=g_{2 l}=0\right\}
$$

on $\mathbb{E}=\mathbb{P}\left(T_{p} \mathbb{P}\right) \cong \mathbb{P}^{M}$. 
Proposition 3.2. For a general (in the sense of Zariski topology) fiber space $V / \mathbb{P}^{1}$ for any smooth point $o \in V \backslash \sigma^{-1}(W)$ the following estimate holds:

$$
\operatorname{deg} \mathbb{L}(o) \leq \lambda_{m, l}=\frac{m !}{6} \frac{(2 l-1) !}{(l-1) !}-1
$$

Proof. We will describe the scheme of arguments and give with all details the main technical lemma which makes it possible to estimate the multiplicities of lines passing through an arbitrary point $o \in V$. The very computations, which are, on one hand, elementary, and on the other hand, tedious, will not be given.

Set

$$
\mathcal{H}=\prod_{i=1}^{m} \mathbb{P}\left(H^{0}\left(\mathbb{P}^{m}, \mathcal{O}_{\mathbb{P}^{M}}(i)\right)\right) \times \prod_{j=l+1}^{2 l} \mathbb{P}\left(H^{0}\left(\mathbb{P}^{M}, \mathcal{O}_{\mathbb{P}^{M}}(j)\right)\right) .
$$

To simplify the notations, we write down a set of non-zero polynomials $\left(q_{\sharp}, g_{\sharp}\right) \in \mathcal{H}$ as

$$
h_{\sharp}=\left(h_{1}, \ldots, h_{M+1}\right) \in \mathcal{H},
$$

where interchanging the direct factors we assume that $\operatorname{deg} h_{i+1} \geq \operatorname{deg} h_{i}$. Let

$$
\mathcal{H}^{+} \subset \mathcal{H}
$$

be the space of all collections $h_{\sharp}$ such that the scheme of common zeros of the polynomials $h_{i}$ is zero-dimensional or empty. For $h_{\sharp} \in \mathcal{H}^{+}$let $\mathbb{L}\left(h_{\sharp}\right)$ be the 0 -cycle of common zeros of the system $h_{\sharp}$. Now we have the following fact.

Proposition 3.3. The codimension of the closed set

$$
\mathcal{H}^{+}(j)=\left\{h_{\sharp} \in \mathcal{H}^{+} \mid \operatorname{deg} \mathbb{L}\left(h_{\sharp}\right) \geq j\right\}
$$

for $j=\lambda_{m, l}+1$ is not less than $M+2$.

Proposition 3.2 follows from this fact automatically. Now we explain how to prove Proposition 3.3. In fact, for $j=\lambda_{m, l}+1$ a much sharper bound for the codimension codim $\mathcal{H}^{+}(j)$ can be obtained in this way than $M+2$ but we do not need that.

First of all, note an obvious fact: for $j=0,1, \ldots, M+2$ the following estimate holds:

$$
\operatorname{codim}\left\{h_{\sharp} \in \mathcal{H}^{+} \mid \sharp \operatorname{Supp} \mathbb{L}\left(h_{\sharp}\right) \geq j\right\}=j .
$$

This implies, that we can assume that the support of the cycle $\mathbb{L}\left(h_{\sharp}\right)$ consists of $j \leq M+1$ distinct points (that is, at most $M+1$ distinct lines pass through any point $o \in V$ in the fiber $F \ni o$ ). However, as the dimension $M$ grow, the multiplicity of these points can be very high. Let us consider the following

Example. Let $p \in S=\left\{h_{1}=\ldots=h_{M-a}=0\right\}$ be a non-singular point, so that $S \ni p$ is a germ of a smooth $a$-dimensional variety. When we require that

$$
\operatorname{mult}_{p}\left\{\left.h_{j}\right|_{S}=0\right\} \geq 2,
$$


$j=M-a+1, \ldots, M+1$, we impose precisely $a+1$ conditions on $h_{j}$. Taking into account that the point $p$ is arbitrary, we obtain

$$
a(a+1) \sim a^{2}
$$

conditions on the polynomials $h_{j}, M-a+1 \leq j \leq M+1$, and these conditions are independent. Thus for $a \sim \sqrt{M}$ there are points on $V$ which satisfy the condition (32). For those points we get

$$
\operatorname{deg} \mathbb{L}(o) \geq 2^{a} \sim 2^{\sqrt{M}} .
$$

This example is a model one showing how the function

$$
\max _{o \in V} \operatorname{deg} \mathbb{L}(o)
$$

grows for a general variety $V / \mathbb{P}^{1}$ as $M \rightarrow \infty$. Note, however, that for small values of $M$ we obtain the same estimate as for the number of distinct lines

$$
\max _{o \in V} \sharp \operatorname{Supp} \mathbb{L}(o) .
$$

Now let us consider the problem of estimating the codimension of the closed set $\mathcal{H}^{+}(j)$. By what was said above, we may make the set $\mathcal{H}^{+}$smaller and assume that

$$
\sharp \operatorname{Supp} \mathbb{L}\left(h_{\sharp}\right) \leq M+1
$$

for any $h_{\sharp} \in \mathcal{H}^{+}$. For each $i=1, \ldots, M$ set

$$
Y_{i}=\left\{h_{1}=\ldots=h_{i}=0\right\}=\underset{j=1}{\stackrel{i}{o}}\left\{h_{j}=0\right\}
$$

to be the algebraic cycle of the scheme-theoretic intersection of the hypersurfaces $\left\{h_{j}=0\right\}, \operatorname{codim}_{\mathbb{E}} Y_{i}=i$. Set also

$$
Y_{M+1}=\left\{h_{1}=\ldots=h_{M-1}=h_{M+1}=0\right\} .
$$

Fix the points $o_{1}, \ldots, o_{k} \in \mathbb{E}, k \leq M+1$ and for each integer-valued matrix

$$
\mathbb{M}=\left\|\mu_{i j}\right\|_{\substack{1 \leq i \leq k, 1 \leq j \leq M+1}} \in \operatorname{Mat}_{k \times(M+1)}\left(\mathbb{Z}_{+}\right)
$$

consider the set

$$
\mathcal{H}(\mathbb{M})=\left\{h_{\sharp} \mid \text { mult }_{o_{i}} Y_{j}=\mu_{i j}\right\} \subset \mathcal{H}^{+} .
$$

(Naturally, it is sufficient to consider only those matrices which satisfy the nondecreasing condition

$$
\mu_{i, 1} \leq \mu_{i, 2} \leq \ldots \leq \mu_{i, M-1} \leq \min \left(\mu_{i, M}, \mu_{i, M+1}\right),
$$


otherwise either $\mathcal{H}(\mathbb{M})$ is empty or the point $o_{i}$ can be removed.) Obviously,

$$
\operatorname{deg} \mathbb{L}\left(h_{\sharp}\right) \leq \sum_{i=1}^{k} \min \left(\mu_{i, M}, \mu_{i, M+1}\right) .
$$

Now to prove Proposition 3.3 we must estimate the codimension

$$
\operatorname{codim}_{\mathcal{H}} \overline{\mathcal{H}(\mathbb{M})}
$$

for all matrices $\mathbb{M}$, satisfying the inequality

$$
\sum_{i=1}^{k} \min \left(\mu_{i, M}, \mu_{i, M+1}\right) \geq \lambda_{m, l}+1 .
$$

Below in Sec. 3.6 we give an inductive method of estimating the codimension $\operatorname{codim} \overline{\mathcal{H}(M)}$ at each step of our procedure of making $Y_{i+1}$ from $Y_{i}$. Now the proof of Proposition 3.3 is completed by tedious but absolutely elementary computations based on Lemma 3.5, which is proved below. We do not give these computations here.

Q.E.D. for Propositions 3.3 and 3.2.

\subsection{A method of estimating the degree}

Let $Y \subset \mathbb{A}=\mathbb{C}^{N}$ be an effective cycle of dimension $a \geq 1, o_{1}, \ldots, o_{k} \in Y$ a set of pair-wise distinct points. Set

$$
\mu_{i}=\operatorname{mult}_{o_{i}} Y \text {, }
$$

$i=1, \ldots, k$. Let $\mathcal{P}_{e}\left(z_{1}, \ldots, z_{N}\right)$ be the space of all (non-homogeneous) polynomials of degree not higher than $e \in \mathbb{Z}_{+}$, in the variables $z_{1}, \ldots, z_{N}$. Let $c_{1}, \ldots, c_{k} \in \mathbb{Z}_{+}$be a set of positive integers such that

$$
c_{1}+\ldots+c_{k-1}+(k-1) \leq e, \quad(k-1) a \leq N
$$

(if $k=1$, then no restrictions are imposed). Set

$$
a_{1}=\ldots=a_{k-1}=a, \quad a_{k}=\min (a, N-(k-1) a),
$$

$c_{i}^{*}=c_{i}$ for $i=1, \ldots, k-1$,

$$
c_{k}^{*}=\min \left(c_{k}, e-c_{1}-\ldots-c_{k-1}-(k-1)\right) .
$$

Let

$$
U_{Y}=\left\{f \in \mathcal{P}_{e}\left(z_{*}\right) \mid \operatorname{dim}\{f=0\} \cap \operatorname{Supp} Y=a-1\right\}
$$

be an (open) set of polynomials that do not vanish identically on each component of the cycle $Y$. Set

$$
U_{c_{*}}=U\left(c_{1}, \ldots, c_{k}\right)=\left\{f \in U_{Y} \mid \operatorname{mult}_{o_{i}}\left\{\left.f\right|_{Y}=0\right\} \geq c_{i} \mu_{i}+1\right\} .
$$


Obviously, the subset $U_{c_{*}} \subset U_{Y}$ is a Zariski closed set.

Lemma 3.5. The following estimate holds:

$$
\operatorname{codim} U_{c_{*}} \geq \Delta\left(c_{1}, \ldots, c_{k}\right)=\sum_{i=1}^{k}\left(\begin{array}{c}
a_{i}+c_{i}^{*} \\
c_{i}^{*}
\end{array}\right) .
$$

Proof. Let $\bar{U}_{c_{*}} \subset \mathcal{P}_{e}\left(z_{*}\right)$ be the closure of the set $U_{c_{*}}$. In order to prove the inequality (33), it is sufficient to produce a closed subset $Z \subset \mathcal{P}_{e}\left(z_{*}\right)$, satisfying the inequality

$$
\operatorname{dim} Z=\Delta\left(c_{1}, \ldots, c_{k}\right)
$$

and such that

$$
Z \cap \bar{U}_{c_{*}}=\{0\}
$$

Obviously, $0 \in \bar{U}_{c_{*}}$, since $\bar{U}_{c_{*}}$ is a cone with the vertex at zero. We first explain how such a set can be constructed in the case when there is only one point $o=o_{1}, k=1$, which we without loss of generality can assume to be the origin $o=(0, \ldots, 0) \in \mathbb{A}$. To simplify our notations, we write

$$
c_{1}=c_{1}^{*}=c \leq e, \quad a_{1}=a \leq N, \quad \mu_{1}=\mu .
$$

Let $\varphi: \widetilde{\mathbb{A}} \rightarrow \mathbb{A}$ be the blow up of the point $o, E=\varphi^{-1}(o) \subset \widetilde{\mathbb{A}}$ the exceptional divisor $E \cong \mathbb{P}^{N-1}$. Consider an effective divisor $D \subset \mathbb{A}, o \in D$, which contains no component of the cycle $Y$, so that

$$
\operatorname{dim}(\operatorname{Supp} Y \cap \operatorname{Supp} D)=a-1
$$

and the effective cycle

$$
Y_{D}=(Y \circ D)
$$

of dimension $a-1$ is well defined. Recall how the multiplicity of this cycle at the point $o$ is computed. Let $\widetilde{Y}, \widetilde{D} \subset \widetilde{\mathbb{A}}$ be the strict transforms of the cycle $Y$ and divisor $D$, respectively. Set

$$
Y_{E}=(\tilde{Y} \circ E)=\sum_{i \in I} b_{i} B_{i}
$$

to be the projectivized tangent cone to $Y$ at the point $o \in \operatorname{Supp} Y$. We get

$$
(\widetilde{D} \circ \tilde{Y})=(\widetilde{D \circ Y})+\sum_{i \in I} d_{i} B_{i}
$$

for some nonnegative $d_{i} \in \mathbb{Z}_{+}$. Now

$$
\operatorname{mult}_{o} Y_{D}=\mu \text { mult }_{o} D+\sum_{i \in I} d_{i} \operatorname{deg} B_{i}
$$

(It is a standard fact of the intersection theory, see [21].) It follows that if $B_{i} \not \subset \widetilde{D}$ for all $i \in I$, then $d_{i}=0$ and thus the following equality holds:

$$
\operatorname{mult}_{o} Y_{D}=\mu \text { mult }_{o} D \text {. }
$$

We say that a set of linear functions $z_{1}, \ldots, z_{a}$ is correct with respect to the pair $(o \in Y)$, if 
- $B_{i} \not \subset \widetilde{\left(z_{1}\right)}$ for all $i \in I$;

- the projection $\pi_{\left(z_{1}, \ldots, z_{a}\right)}: \mathbb{C}^{N} \rightarrow \mathbb{C}^{a}$ is dominant on each irreducible component of the cycle $Y$;

- the following infinitesimal condition is satisfied at the point $o$. Taking $\left(z_{1}: \ldots: z_{N}\right)$ for homogeneous coordinates on $E$, consider the affine set $\left\{z_{1} \neq 0\right\}$ with the coordinates $\left(y_{2}, \ldots, y_{N}\right)$, where $y_{i}=z_{i} / z_{1}$. The collection of functions $\left(y_{2}, \ldots, y_{a}\right)$ determines a projection $\iota: \mathbb{C}^{N-1} \rightarrow \mathbb{C}^{a-1}$. Now our condition is formulated in the following way: the restriction

$$
\iota_{B_{i}}: B_{i} \cap\left\{z_{1} \neq o\right\} \rightarrow \mathbb{C}^{a-1}
$$

of the projection $\iota$ onto each component $B_{i}$ is a dominant map.

It is easy to see that a general set $\left(z_{1}, \ldots, z_{a}\right)$ of linear functions is correct with respect to the pair $(o \in Y)$. By the definition of correctness for any non-zero polynomial $f \in \mathcal{P}_{e}\left(z_{i}, \ldots, z_{a}\right)$ in the variables $z_{1}, \ldots, z_{a}$ we get

$$
B_{i} \not \subset \widetilde{(f)}
$$

and thus by what was said above mult $Y_{(f)} \leq \mu \operatorname{deg} f$. Thus we can set

$$
Z=\mathcal{P}_{c}\left(z_{1}, \ldots, z_{a}\right) .
$$

For any non-zero polynomial $f \in Z$ the set $\{f=0\}$ contains entirely none of the irreducible components of the cycle $Y$, so that $Z \backslash\{0\} \subset U_{Y}$. Therefore $Z \cap \bar{U}_{c}=\{0\}$, as required.

Now let us consider the general case of an arbitrary $k \geq 2$. Here we have $k$ points $o_{1}, \ldots, o_{k} \in \operatorname{Supp} Y$. Consider a system of affine functions

$$
\begin{array}{ccc}
l_{1,1}, & \ldots, & l_{1, a_{1}}, \\
& \ldots & \\
l_{k, 1}, & \ldots, & l_{k, a_{k}},
\end{array}
$$

$a_{1}=\ldots=a_{k-1}=a, a_{k}=\min (a, N-(k-1) a)$, satisfying the following conditions:

- the linear parts of the affine functions $l_{*, *}$ are linear independent, that is, form a part of a basis of the space $\mathbb{C}^{N}$;

- for any $i \in\{1, \ldots, k-1\}$ we have $l_{i, \alpha}\left(o_{i}\right)=0$ and the system of functions $\left(l_{i, 1}, \ldots, l_{i, a}\right)$ is correct with respect to the pair $\left(o_{i} \in Y\right) ; l_{k, \alpha}\left(o_{k}\right)=0$ and the system of functions $\left(l_{k, 1}, \ldots, l_{k, a_{k}}\right)$ is a part of a correct set for the pair $\left(o_{k} \in Y\right)$;

- for $i \neq j$ we have $l_{i, 1}\left(o_{j}\right) \neq 0$. 
Now set

$$
Z=\sum_{i=1}^{k}\left(\prod_{j=1}^{i-1} l_{j, 1}^{c_{j}+1}\right) \mathcal{P}_{c_{i}^{*}}\left(l_{i, 1}, \ldots, l_{i, a_{i}}\right),
$$

where $\mathcal{P}_{\alpha}(\sharp)$ denotes the linear space of all polynomial functions of degree $\alpha$ in the affine functions $\sharp$. Note that the sum in (35) is direct, since the linear parts of the functions $l_{\sharp}$ are linear independent.

Lemma 3.6. The closed algebraic subsets $Z$ and $\bar{U}_{c_{*}}$ intersect each other by zero only.

Proof. For $f \in Z$ we have the decomposition

$$
f=f_{1}+\ldots+f_{k}, \quad f_{i} \in Z_{i}=\left(\prod_{j=1}^{i-1} l_{j, 1}^{c_{j}+1}\right) \mathcal{P}_{c_{i}^{*}}\left(l_{i, 1}, \ldots, l_{i, a_{i}}\right)
$$

which is uniquely determined since $Z=\bigoplus_{i=1}^{k} Z_{i}$.

By construction, for each $i \in\{1, \ldots, k\}$ we have

$$
\operatorname{mult}_{o_{j}}\left\{\left.f_{i}\right|_{Y}=0\right\} \geq\left(c_{j}+1\right) \mu_{j} \geq c_{j} \mu_{j}+1
$$

for all $j \leq i-1$. In particular, if $f \in Z \cap \bar{U}_{c_{*}}$, then the following estimate holds:

$$
\operatorname{mult}_{o_{1}}\left\{\left.f_{1}\right|_{Y}=0\right\} \geq c_{1} \mu_{1}+1
$$

since for $f_{2}, \ldots, f_{k}$ the estimate (36) with $j=1$ is satisfied. Arguing as above, we conclude that $f_{1} \equiv 0$. Assume that it is already proved that

$$
f_{1} \equiv \ldots \equiv f_{\gamma} \equiv 0
$$

Since for $j=\gamma+1$ the estimate (36) holds for all $i \geq \gamma+2$, we conclude that

$$
\operatorname{mult}_{o_{\gamma+1}}\left\{\left.f_{\gamma+1}\right|_{Y}=0\right\} \geq c_{\gamma+1} \mu_{\gamma+1}+1 .
$$

However by construction

$$
f_{\gamma+1}=\left(\prod_{j=1}^{\gamma} l_{j, 1}^{c_{j}+1}\right) f_{\gamma+1}^{\sharp},
$$

where $f_{\gamma+1}^{\sharp} \in \mathcal{P}_{c_{\gamma+1}^{*}}\left(l_{\gamma+1,1}, \ldots, l_{\gamma+1, a_{\gamma+1}}\right)$. Taking into account that for $j=1, \ldots, \gamma$ we have $l_{j, 1}\left(o_{\gamma+1}\right) \neq 0$, we see that the estimate (37) remains valid if $f_{\gamma+1}$ is replaced by $f_{\gamma+1}^{\sharp}$. Now arguing like in the case $k=1$ above, we conclude that $f_{\gamma+1} \equiv 0$. Q.E.D. for Lemma 3.6.

Taking into account that the equality (34) holds (this is obvious from the explicit construction of the linear space $Z(\underline{35})$ ), we complete the proof of Lemma 3.5. 


\section{References}

1. Brown G., Corti A. and Zucconi F. Birational geometry of 3-fold Mori fibre spaces. Preprint, 2003, 40 p. arXiv: math.AG/0307301.

2. Corti A., Pukhlikov A. and Reid M., Fano 3-fold hypersurfaces, in "Explicit Birational Geometry of Threefolds", London Mathematical Society Lecture Note Series 281 (2000), Cambridge University Press, 175-258.

3. Grinenko M.M., Birational automorphisms of a three-dimensional double cone. Sbornik: Mathematics. 189 (1998), no. 7, 37-52.

4. Grinenko M.M., Birational properties of pencils of del Pezzo surfaces of degrees 1 and 2. Sbornik: Mathematics. 191 (2000), no. 5, 17-38.

5. Grinenko M.M., Birational properties of pencils of del Pezzo surfaces of degrees 1 and 2. II. Sbornik: Mathematics. 194 (2003).

6. Iskovskikh V.A. and Manin Yu.I., Three-dimensional quartics and counterexamples to the Lüroth problem, Math. USSR Sb. 86 (1971), no. 1, 140-166.

7. Pukhlikov A.V., Birational automorphisms of three-dimensional algebraic varieties with a pencil of del Pezzo surfaces, Izvestiya: Mathematics 62:1 (1998), 115-155.

8. Pukhlikov A.V., Birational automorphisms of Fano hypersurfaces, Invent. Math. 134 (1998), no. 2, 401-426.

9. Pukhlikov A.V., Birationally rigid Fano double hypersurfaces, Sbornik: Mathematics 191 (2000), No. 6, 101-126.

10. Pukhlikov A.V., Birationally rigid Fano fibrations, Izvestiya: Mathematics 64 (2000), 131-150.

11. Pukhlikov A.V., Certain examples of birationally rigid varieties with a pencil of double quadrics. Journal of Math. Sciences. 1999. V. 94, no. 1, 986-995.

12. Pukhlikov A.V., Birational automorphisms of algebraic varieties with a pencil of double quadrics. Mathematical Notes. 67 (2000), 241-249.

13. Pukhlikov A.V., Essentials of the method of maximal singularities, in "Explicit Birational Geometry of Threefolds", London Mathematical Society Lecture Note Series 281 (2000), Cambridge University Press, 73-100.

14. Pukhlikov A.V., Birationally rigid Fano complete intersections, Crelle J. für die reine und angew. Math. 541 (2001), 55-79.

15. Pukhlikov A.V., Birationally rigid iterated Fano double covers. Izvestiya: Mathematics. 67 (2003), no. 3, 555-596.

16. Pukhlikov A.V., Birationally rigid varieties with a pencil of Fano double covers. I. Sbornik: Mathematics 195 (2004). Available as a preprint MPI-2003, no. 102.

17. Sarkisov V.G., Birational automorphisms of conic bundles, Izv. Akad. Nauk SSSR, 
Ser. Mat. 44 (1980), no. 4, 918-945 (English translation: Math. USSR Izv. 17 (1981), 177-202).

18. Sarkisov V.G., On conic bundle structures, Izv. Akad. Nauk SSSR, Ser. Mat. 46 (1982), no. 2, 371-408 (English translation: Math. USSR Izv. 20 (1982), no. 2, 354-390).

19. Sobolev I. V., On a series of birationally rigid varieties with a pencil of Fano hypersurfaces. Mat. Sb. 192 (2001), no. 10, 123-130 (English translation in Sbornik: Math. 192 (2001), no. 9-10, 1543-1551).

20. Sobolev I. V., Birational automorphisms of a class of varieties fibered into cubic surfaces. Izv. Ross. Akad. Nauk Ser. Mat. 66 (2002), no. 1, 203-224.

21. Fulton W., Intersection Theory, Springer-Verlag, 1984.

e-mail: pukh@mi.ras.ru, pukh@liv.ac.uk 